\title{
Variational Analysis of the Coupling Between a Geometrically Exact Cosserat Rod and an Elastic Continuum *
}

\author{
Oliver Sander and Anton Schiela
}

September 24, 2012

\begin{abstract}
We formulate the static mechanical coupling of a geometrically exact Cosserat rod to a nonlinearly elastic continuum. In this setting, appropriate coupling conditions have to connect a one-dimensional model with director variables to a three-dimensional model without directors. Two alternative coupling conditions are proposed, which correspond to two different configuration trace spaces. For both we show existence of solutions of the coupled problems, using the direct method of the calculus of variations. From the first-order optimality conditions we also derive the corresponding conditions for the dual variables. These are then interpreted in mechanical terms.
\end{abstract}

AMS MSC 2000: 74K10, 74B20, 49K20

Keywords: coupling conditions, energy minimization, Cosserat rod, hyperelastic material

\section{Introduction}

In the mechanical simulation of large structures frequently the structure to be simulated combines long slender parts together with bulky ones. A typical example would be a suspension bridge, with bulky pillars and slender cables. Discretizing such a heterogeneous structure with a single finite element mesh is prohibitively expensive, because the usual shape regularity assumptions require a large amount of elements in the slender parts. As an alternative, structural mechanics has invented reduced models, which are mathematically oneor two-dimensional objects, and which allow to model slender structures more efficiently. There is quite a variety of such models. They may be linear or geometrically exact, and may or may not comprise certain orientation or director variables. A structure combining bulky and slender parts could be modeled by a combination of full-dimensional continuum and lower-dimensional reduced models. Berlin 
The problem then arises as to how to couple the lower-dimensional models with the bulk model. Coupling conditions need to be formulated, which have to be adapted to the continuum and reduced model. In particular, if lower-dimensional models with directors are involved, coupling conditions for the director variables are needed. Coupling conditions can be derived ad hoc from physical intuition. However, for a mathematically rigorous theory wellposedness of the coupled problem has to be shown. By this we mean primarily the existence of solutions. Uniqueness of these solutions can only be expected as far as the bulk and reduced models themselves allow for unique solutions.

In this article we focus on the coupling of a three-dimensional continuum model to a geometrically exact one-dimensional model with an orthonormal director frame, a so-called Cosserat rod [1. We assume that both objects are governed by hyperelastic material laws, and consider the static case only.

We then propose two different coupling conditions and show that the coupled problem has solutions for both of them. Our existence proof uses the direct method of the calculus of variations. From the optimality criteria we also obtain the corresponding dual conditions. These dual conditions can be interpreted physically in terms of forces and moments transmitted at the interface.

Existence of more than one set of plausible coupling conditions is a general feature of heterogeneous models (cf. 4] for the linear case without directors). Indeed, coupling conditions need to be formulated in an interface space, which is usually the trace space of the submodel configuration at the interface. When coupling two instances of the same model across a common boundary, these trace spaces coincide. This common trace space is then the canonical choice of interface space to formulate the coupling conditions in.

The situation is more complicated when the two models differ in their kinematics. There are then two different trace spaces leading to two different families of coupling conditions, which are both equally valid. In their treatment of the coupling between linear elastic continua and beams, Blanco et al. 4 even introduce an entire continuum of conditions by interpolating between the two trace spaces. They note, though, that no truly new coupling conditions are produced in this way.

The presence of orientation degrees of freedom in the reduced model adds another set of difficulties. These orientations have no counterpart on the continuum side to couple to. Local rotations of a continuum obtained by polar decomposition (microrotations) are a natural choice, but are difficult to work with in combination with averaging (see 18, and Section 4.3). In this article we choose a different approach which avoids the use of microrotations.

The coupling of mechanical models of differing dimensions has been treated both in the engineering and the mathematical literature. Monaghan et al. 16] describe a $3 \mathrm{~d}-1 \mathrm{~d}$ coupling between linear elastic elements in the discrete setting. Lagnese et al. 15. have studied the coupling of beams to plates extensively. In their work, however, the main focus is on the linearized equations. Modeling of $3 \mathrm{~d}-2 \mathrm{~d}$ junctions between linear elastic objects of different dimensions using a method of asymptotic expansion has been carried out by Ciarlet et al. [7]. Formaggia et al. 12 couple $3 \mathrm{~d}$ and $1 \mathrm{~d}$ variants of the Navier-Stokes equations in a simulation of blood circulation.

As systematic treatment for coupling linear models of different dimensions has been given by Blanco et al. 4. In particular, they provide existence and uniqueness of solutions for their coupled problem. To the knowledge of the 
authors, coupling conditions for a reduced model with director variables have only been treated in Sander [18, 19. There, coupling conditions were derived by a heuristic dimensional reduction. An algorithm based on fixed-point iteration was proposed to numerically solve heterogeneous coupling problems. Our approach here instead suggests to treat the problem as a global minimization problem with nonlinear constraints. A detailed treatment may appear in a separate article.

We proceed as follows. In Chapters 2 and 3 we formally introduce the rod and continuum models. Then, in Chapter 4 we propose two sets of coupling conditions, which differ in the choice of interface space. In Chapter 5 we prove existence of solutions for both of these conditions. Assuming some additional regularity, in Chapter 6 we derive coupling conditions for the dual variables from the optimality conditions of the minimization problem. In the final Chapter 7 these are interpreted in terms of physical quantities such as coupling forces and torques.

\section{Geometrically Exact Cosserat Rods}

In this section we briefly present Cosserat rods, which model the large deformation behavior of long, slender objects. For an in-depth presentation see the book by Antman [1].

\subsection{Rigid Body Motion}

Consider $\mathbb{R}^{3}$, equipped with the Euclidean scalar product $v, w \mapsto v \cdot w$ and the cross product $v, w \mapsto v \times w$. Let $\mathrm{SO}(3)$ be the special orthogonal group in $\mathbb{R}^{3}$, that is the group of orthogonal $3 \times 3$-matrices with positive determinant. Elements $R$ of $\mathrm{SO}(3)$ act on $\mathbb{R}^{3}$ by rotation around the origin.

Consider the product space $\mathbb{R}^{3} \times \mathrm{SO}(3)$. We denote elements of this space as tuples $\rho=(r, R)$. Together with the product

$$
\left(r_{1}, R_{1}\right) \cdot\left(r_{2}, R_{2}\right):=\left(R_{1} r_{2}+r_{1}, R_{1} R_{2}\right),
$$

this space becomes the special Euclidean group

$$
\mathrm{SE}(3)=\mathbb{R}^{3} \rtimes \mathrm{SO}(3) .
$$

An element $\rho=(r, R) \in \mathrm{SE}(3)$ acts on $\mathbb{R}^{3}$ by a rigid body motion $\rho: x \mapsto R x+r$, and the group multiplication corresponds to a composition of these motions. The inverse of a group element $(r, R)$ is

$$
(r, R)^{-1}=\left(-R^{-1} r, R^{-1}\right) .
$$

The group $\mathrm{SO}(3)$ has the structure of a three-dimensional compact $C^{\infty}$ manifold. For any $R \in \mathrm{SO}(3)$ the tangent space of $\mathrm{SO}(3)$ at $R$ (which is a threedimensional linear space) can be characterized in two different ways, namely

$$
T_{R} \mathrm{SO}(3)=\left\{\delta R \in \mathbb{R}^{3 \times 3} \mid \delta R=\mathbf{U} R, \mathbf{U} \in \mathfrak{s o}(3)\right\},
$$

which is called the spatial representation, and

$$
T_{R} \mathrm{SO}(3)=\left\{\delta R \in \mathbb{R}^{3 \times 3} \mid \delta R=R \mathrm{U}, \mathrm{U} \in \mathfrak{s o}(3)\right\},
$$


which is called the body representation. The Lie algebra $\mathfrak{s o}(3)$ of $\mathrm{SO}(3)$ is the space of skew-symmetric $3 \times 3$ matrices. Obviously, we have the relation

$$
\mathbf{U}=R \mathrm{U} R^{-1},
$$

and hence $\mathbf{U}$ arises from $\mathbf{U}$ by an orthogonal change of coordinates. Both can be interpreted as infinitesimal rotations.

With each $U \in \mathfrak{s o}(3)$ we associate a vector $u \in \mathbb{R}^{3}$ via the relation

$$
U v=u \times v \quad \forall v \in \mathbb{R}^{3},
$$

and we will denote this relation (which is in fact a linear isomorphism) by $U=u^{\times}$, with the inverse $u=U_{\times}$. The vector $u$ is called the axial vector of the skew-symmetric matrix $U$.

A combination of the above results yields a linear isomorphism that identifies $T_{R} \mathrm{SO}(3)$ with $\mathbb{R}^{3}$ via

$$
\begin{aligned}
\boldsymbol{\Phi}_{R}: T_{R} \mathrm{SO}(3) & \rightarrow \mathbb{R}^{3} \\
\delta R & \mapsto \mathbf{u}:=\left(\delta R R^{-1}\right)_{\times},
\end{aligned}
$$

and we call $\mathbf{u}$ the spatial vector of $\delta R$. Similarly, we have an isomorphism

$$
\begin{aligned}
\Phi_{R}: T_{R} \mathrm{SO}(3) & \rightarrow \mathbb{R}^{3} \\
\delta R & \mapsto \mathrm{u}:=\left(R^{-1} \delta R\right)_{\times},
\end{aligned}
$$

where $\mathrm{u}$ is called the body vector of $\delta R$. A short computation that uses the relation $R(w \times v)=R w \times R v$ for $R \in \mathrm{SO}(3)$ yields

$$
\mathbf{u}=R \mathbf{u} .
$$

When working with axial vectors, the well-known identity of cyclic permutation

$$
u \cdot(v \times w)=v \cdot(w \times u)=w \cdot(u \times v)
$$

for vectors $u, v, w \in \mathbb{R}^{3}$ is a useful tool.

The cotangent space $T_{R} \mathrm{SO}(3)^{*}$ of $\mathrm{SO}(3)$ at $R$ is the space of linear functionals on $T_{R} \mathrm{SO}(3)$. The adjoint mappings $\boldsymbol{\Phi}_{R}^{*}:\left(\mathbb{R}^{3}\right)^{*} \rightarrow T_{R} \mathrm{SO}(3)^{*}$ and $\Phi_{R}^{*}:\left(\mathbb{R}^{3}\right)^{*} \rightarrow$ $T_{R} \mathrm{SO}(3)^{*}$ are isomorphisms, so that every linear functional $B^{*} \in T_{R} \mathrm{SO}(3)^{*}$ has a spatial representation $\mathbf{m}^{*}$ and a body representation $\mathrm{m}^{*}$ by

$$
B^{*}=\boldsymbol{\Phi}_{R}^{*} \mathbf{m}^{*}=\Phi_{R}^{*} \mathrm{~m}^{*}
$$

This means, for example, that

$$
B^{*}(\delta R)=\left(\boldsymbol{\Phi}_{R}^{*} \mathbf{m}^{*}\right)(\delta R)=\mathbf{m}^{*}\left(\boldsymbol{\Phi}_{R} \delta R\right)=\mathbf{m}^{*}\left(\left(\delta R R^{-1}\right)_{\times}\right)
$$

for any $\delta R \in T_{R} \mathrm{SO}(3)$. Finally, we can associate to $\mathbf{m}^{*} \in\left(\mathbb{R}^{3}\right)^{*}$ a vector $\mathbf{m} \in \mathbb{R}^{3}$ via $\mathbf{m} \cdot \mathbf{u}=\mathbf{m}^{*}(\mathbf{u})$ for all $\mathbf{u} \in \mathbb{R}^{3}$. Similarly, we can define a vector $m$ associated to $\mathrm{m}^{*}$ via $\mathbf{m} \cdot \mathbf{u}=\mathrm{m}^{*}(\mathbf{u})$ and compute, using $\mathbf{m} \cdot \mathbf{u}=\mathbf{m} \cdot \mathbf{u}$, Equation (3), and $R^{-1}=R^{T}$, that

$$
\mathbf{m}=R \mathbf{m} .
$$




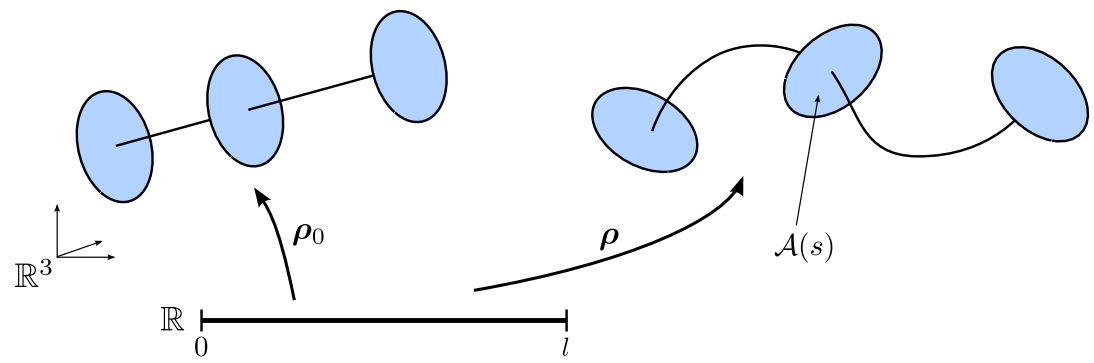

Figure 1: Kinematics of Cosserat rods. Under deformation, rod cross-sections remain planar, but not necessarily orthogonal to the centerline.

The structure of the tangent spaces of $\mathrm{SE}(3)$ can be inferred from the general rules about tangent spaces of product manifolds. For each $\rho=(r, R) \in \operatorname{SE}(3)$ we have

$$
T_{\rho} \mathrm{SE}(3)=T_{r} \mathbb{R}^{3} \times T_{R} \mathrm{SO}(3) .
$$

By the above identifications, each element $(\delta r, \delta R) \in T_{\rho} \mathrm{SE}(3)$ has a representation $(\mathbf{v}, \mathbf{u})=\left(\delta r, \boldsymbol{\Phi}_{R} \delta R\right)$ in spatial coordinates and $(\mathbf{v}, \mathbf{u})=\left(R^{-1} \delta r, \Phi_{R} \delta R\right)$ in body coordinates. Clearly, $(\mathbf{v}, \mathbf{u})=(R \mathbf{v}, R \mathbf{u})$. Note that the factor $R^{-1}$ in the definition of $v$ originates from the action of SE(3) on its own tangent bundle.

For the cotangent space $T_{\rho} \mathrm{SE}(3)^{*}$ we have analogously

$$
T_{\rho} \mathrm{SE}(3)^{*}=\left(T_{r} \mathbb{R}^{3}\right)^{*} \times T_{R} \mathrm{SO}(3)^{*} .
$$

Each element $\left(b^{*}, B^{*}\right) \in T_{\rho} \mathrm{SE}(3)^{*}$ has a representation $\left(\mathbf{n}^{*}, \mathbf{m}^{*}\right)=\left(b^{*}, \boldsymbol{\Phi}_{R}^{-*} B^{*}\right)$ in spatial coordinates and $\left(\mathrm{n}^{*}, \mathrm{~m}^{*}\right)=\left(R^{*} b^{*}, \Phi_{R}^{-*} B^{*}\right)$ in body coordinates, where $\Phi_{R}^{-*}:=\left(\Phi_{R}^{*}\right)^{-1}$. Again, $(\mathbf{n}, \mathbf{m})=(R \mathrm{n}, R \mathrm{~m})$.

\subsection{Static Rod Model}

The theory of Cosserat rods views a rod as a curve in space, with a planar cross-section attached at each point. The central assumption is that under load, cross-sections do not change shape. They may, however, change their orientations, and are in particular not restricted to remain normal to the curve tangent vector (Figure 1). Hence, configurations of Cosserat rods are continuous maps

$$
\boldsymbol{\rho}:[0, l] \rightarrow \mathrm{SE}(3), \quad s \mapsto \boldsymbol{\rho}(s)=(r(s), R(s)),
$$

for some parameter interval $[0, l]$. While the first component $r(s) \in \mathbb{R}^{3}$ of $\boldsymbol{\rho}(s)$ determines the position of the center curve of the rod at $s$, the second component $R(s) \in \mathrm{SO}(3)$ determines the orientation of the cross-section $\mathcal{A}(s)$ (Figure 1).

We single out one configuration function $\rho_{0}:[0, l] \rightarrow \mathrm{SE}(3)$ and call it the reference configuration. It will be convenient (but not necessary) to choose $\boldsymbol{\rho}_{0}$ to be the stress-free configuration.

Let $\boldsymbol{\rho}:[0, l] \rightarrow \mathrm{SE}(3)$ be a given rod configuration. We define the derivative

$$
\boldsymbol{\rho}^{\prime}:[0, l] \rightarrow T \mathrm{SE}(3), \quad s \mapsto\left(r^{\prime}(s), R^{\prime}(s)\right),
$$


with respect to $s$, and the spatial strains

$$
(\mathbf{v}, \mathbf{u}):=\left(r^{\prime}, \mathbf{\Phi}_{R} R^{\prime}\right) .
$$

Correspondingly, we write $\left(\mathbf{v}_{0}, \mathbf{u}_{0}\right)$ for the spatial strain of the reference configuration $\boldsymbol{\rho}_{0}$. The strains become invariant under rigid-body motions when expressed in body coordinates. Using (3), we get $(\mathrm{v}, \mathrm{u}) \in \mathbb{R}^{3} \times \mathbb{R}^{3}$ with

$$
(\mathrm{v}, \mathrm{u})=\left(R^{-1} r^{\prime}, \Phi_{R} R^{\prime}\right)=\left(R^{-1} \mathbf{v}, R^{-1} \mathbf{u}\right),
$$

and in particular the reference strains $\left(\mathrm{v}_{0}, \mathrm{u}_{0}\right)=\left(R_{0}^{-1} \mathbf{v}_{0}, R_{0}^{-1} \mathbf{u}_{0}\right)$.

The coefficients of $v$ and $u$ can be interpreted in a natural way. The two values $v_{1}, v_{2}$ are the shear strains, and $v_{3}$ is the stretching strain. Further, the values $\mathrm{u}_{1}, \mathrm{u}_{2}$-infinitesimal rotations about axes in the cross section - are the bending strain, and $\mathrm{u}_{3}$ is the strain related to torsion (cf. [14]).

Physically, the stress variables in an elastic Cosserat rod model are the total resultant force $\mathrm{n}$ and moment $\mathrm{m}$ across a cross-section. Mathematically they are elements of the cotangent bundle of $\mathrm{SE}(3)$, related to the force and moment vectors by the relations $\boldsymbol{\Phi}_{R}^{*}$ and $\boldsymbol{\Phi}_{R}^{*}$. Forces and moments are linked to the strain by constitutive relations which describe the properties of specific materials. We assume that the rod material is hyperelastic in the sense that there exists an energy functional $W: \mathbb{R}^{3} \times \mathbb{R}^{3} \rightarrow \mathbb{R}$ such that the body stresses $\mathrm{n}^{*}, \mathrm{~m}^{*}$ are given by a Legendre transform

$$
\mathrm{n}^{*}=\frac{\partial W}{\partial \mathrm{v}}(\mathrm{v}, \mathrm{u}), \quad \mathrm{m}^{*}=\frac{\partial W}{\partial \mathrm{u}}(\mathrm{v}, \mathrm{u}) .
$$

We assume the strain-energy function $W$ to be convex, Fréchet-differentiable, and coercive in the sense that

$$
\frac{W(w, z)}{|w|^{2}+|z|^{2}} \geq \alpha \quad \text { as } \quad|w|^{2}+|z|^{2} \rightarrow \infty
$$

for some fixed $\alpha>0$.

The coefficients of the body stresses $n$ and $m$ have again a physical interpretation. We refer to $m_{1}, m_{2}$ as the bending moments and to $m_{3}$ as the twisting moment. The values $\mathrm{n}_{1}, \mathrm{n}_{2}$ are shear forces and $\mathrm{n}_{3}$ is the tension.

\subsection{Formulation as a Minimization Problem}

The stable equilibrium configurations of a Cosserat rod with a hyperelastic material law can be characterized as the minima of an energy functional

$$
j: \boldsymbol{\rho} \mapsto \int_{[0, l]} W(\mathrm{v}(\boldsymbol{\rho}), \mathrm{u}(\boldsymbol{\rho})) d s,
$$

where for simplicity we have assumed absence of external volumes forces and moments. To discuss the well-posedness of such minimization problems we need to introduce Sobolev spaces for functions with values in $\operatorname{SE}(3)$. Note that $\operatorname{SE}(3)$ arises naturally as a submanifold of $\mathbb{R}^{3} \times \mathbb{R}^{3 \times 3}$. We define the manifold-valued Sobolev space

$$
H^{1}([0, l], \operatorname{SE}(3)):=\left\{\boldsymbol{\rho} \in H^{1}\left([0, l], \mathbb{R}^{3} \times \mathbb{R}^{3 \times 3}\right) \mid \boldsymbol{\rho}(s) \in \mathrm{SE}(3) \text { a.e. }\right\} .
$$


By [17, Thm. (6)], it is a Hilbert manifold. By the same theorem, tangent vectors at a configuration $\boldsymbol{\rho}$ in the Hilbert manifold $H^{1}([0, l], \mathrm{SE}(3))$ correspond to tangent vector fields along $\boldsymbol{\rho}$ in $\mathrm{SE}(3)$ :

Lemma 2.1. For any given $\boldsymbol{\rho} \in H^{1}([0, l], \mathrm{SE}(3))$ we have

$$
T_{\boldsymbol{\rho}} H^{1}([0, l], \mathrm{SE}(3)):=\left\{\delta \boldsymbol{\rho} \in H^{1}\left([0, l], \mathbb{R}^{3} \times \mathbb{R}^{3 \times 3}\right) \mid \delta \boldsymbol{\rho}(s) \in T_{\boldsymbol{\rho}(s)} \mathrm{SE}(3) \text { a.e. }\right\} .
$$

Since the domain $[0, l]$ is one-dimensional, there is a continuous Sobolev embedding

$$
H^{1}([0, l], \mathrm{SE}(3)) \hookrightarrow C([0, l], \mathrm{SE}(3)),
$$

see, e.g., 3 for a proof. Further, we have the following density result

$$
C^{\infty}([0, l], \mathrm{SE}(3)) \hookrightarrow^{d} H^{1}([0, l], \mathrm{SE}(3))
$$

(cf. [3, 17]).

By our coercivity assumptions on $W$, the space $H^{1}([0, l], \mathrm{SE}(3))$ is the appropriate framework for considering minimization problems for the functional $j$.

Lemma 2.2. Let $W$ be coercive in the sense of (6). Then $j$ is coercive as a function of $(\mathrm{u}, \mathrm{v}) \in L_{2}\left([0, l], \mathbb{R}^{3} \times \mathbb{R}^{3}\right)$, and weakly lower semi-continuous in the space $H^{1}([0, l], \mathrm{SE}(3))$.

Proof. Coercivity of $j$ in $L_{2}\left([0, l], \mathbb{R}^{3} \times \mathbb{R}^{3}\right)$ follows directly from (6). In 20 sequential weak lower semi-continuity in the space $L^{1}\left([0, l], \mathbb{R}^{3} \times \mathbb{R}^{3}\right)$ of strains is shown, which implies weak lower semi-continuity in $L^{2}\left([0, l], \mathbb{R}^{3} \times \mathbb{R}^{3}\right)$. This corresponds to weak lower semicontinuity in $H^{1}([0, l], \mathrm{SE}(3))$.

If we impose Dirichlet boundary conditions

$$
\boldsymbol{\rho}(0)=\boldsymbol{\rho}_{D, 0} \quad \text { and } \quad \boldsymbol{\rho}(l)=\boldsymbol{\rho}_{D, l},
$$

and a corresponding constraint manifold

$$
H_{D, 0, l}^{1}([0, l], \operatorname{SE}(3)):=\left\{\boldsymbol{\rho} \in H^{1}([0, l], \mathrm{SE}(3)) \mid \boldsymbol{\rho}(0)=\boldsymbol{\rho}_{D, 0}, \boldsymbol{\rho}(l)=\boldsymbol{\rho}_{D, l}\right\},
$$

then the problem of finding stable equilibrium configurations of Cosserat rods can be written as the optimization problem

$$
\text { minimize } j \text { in } H_{D, 0, l}^{1}([0, l], \mathrm{SE}(3)) \text {. }
$$

Existence of solutions to this problem has been shown by Seidman and Wolfe [20]. These solutions are generally not unique. However, the following regularity result holds:

Theorem 2.1 ([20], Thm.4.24). Let $(\mathbf{u}(\boldsymbol{\rho}), \mathbf{v}(\boldsymbol{\rho}))$ be a solution of the minimization problem (7), with the boundary conditions $(9)$. Then $(\mathbf{u}(\boldsymbol{\rho}), \mathbf{v}(\boldsymbol{\rho}))$ is in $\left(C^{1}[0,1]\right)^{6}$. 


\subsection{Weak formulation and Neumann-type boundary con- ditions}

We now want to investigate Neumann-type boundary conditions in a variational form. These appear as additional terms in the Euler-Lagrange equations of the energy functional (7).

Let us, however, recall first the well-known case of Dirichlet boundary conditions on both ends (cf., e.g., 6, 20]). Let $Y_{\boldsymbol{\rho}, D, 0, l}$ be the subspace of $T_{\boldsymbol{\rho}} H^{1}([0, l], \mathrm{SE}(3))$ where $\delta \boldsymbol{\rho}(0)=0$ and $\delta \boldsymbol{\rho}(l)=0$ holds in the sense of traces. Then solutions to the minimization problem (11) solve the weak formulation

$$
\boldsymbol{\rho} \in H_{D, 0, l}^{1}([0, l], \mathrm{SE}(3)): \quad 0=T_{\boldsymbol{\rho}} j \delta \boldsymbol{\rho} \quad \text { for all } \delta \boldsymbol{\rho} \in Y_{\boldsymbol{\rho}, D, 0, l},
$$

where the prescribed Dirichlet values are incorporated into the configuration space (10). Explicit computation of $T_{\rho} j \delta \rho$ and integration by parts yields the well-known strong equilibrium equations of forces and moments

$$
\begin{aligned}
\mathbf{n}^{\prime} & =0, & & \text { on }[0, l], \\
\mathbf{m}^{\prime}+r^{\prime} \times \mathbf{n} & =0, & & \text { on }[0, l] .
\end{aligned}
$$

We now assume that the rod is clamped only at the far end $s=l$, but free to move at $s=0$. We introduce the rod Neumann boundary $\gamma:=\{0\} \subset \partial[0, l]$, and the modified configuration and test function spaces

$$
\begin{aligned}
H_{D, l}^{1}([0, l], \mathrm{SE}(3)) & :=\left\{\boldsymbol{\rho} \in H^{1}([0, l], \mathrm{SE}(3)) \mid \boldsymbol{\rho}(l)=\boldsymbol{\rho}_{D, l}\right\} \\
Y_{\boldsymbol{\rho}, D, l} & :=\left\{T_{\boldsymbol{\rho}} H^{1}([0, l], \mathrm{SE}(3)) \mid \delta \boldsymbol{\rho}(l)=0\right\} .
\end{aligned}
$$

We assume that external loads act on the rod boundary $\gamma$, given by the linear functional

$$
\beta \in T_{\left.\boldsymbol{\rho}\right|_{\gamma}} \mathrm{SE}(3)^{*}: \quad(\delta r, \delta R) \mapsto b^{*}(\delta r)+B^{*}(\delta R) .
$$

Subtracting this functional from the weak formulation 12 , we obtain the new problem

$$
\boldsymbol{\rho} \in H_{D, l}^{1}([0, l], \operatorname{SE}(3)): \quad 0=T_{\boldsymbol{\rho}} j \delta \boldsymbol{\rho}-\beta\left(\left.\delta \boldsymbol{\rho}\right|_{\gamma}\right) \quad \text { for all } \delta \boldsymbol{\rho} \in Y_{\boldsymbol{\rho}, D, l} .
$$

The following result shows that the additional term can really be interpreted as a Neumann boundary condition at $s=0$.

Theorem 2.2. Let $\boldsymbol{\rho} \in H_{D, l}^{1}([0, l], \mathrm{SE}(3))$ be a rod configuration such that

$$
0=T_{\boldsymbol{\rho}} j \delta \boldsymbol{\rho}-\beta\left(\left.\delta \boldsymbol{\rho}\right|_{\gamma}\right)
$$

for all admissible variations $\delta \boldsymbol{\rho}=(\delta r, \delta R) \in Y_{\boldsymbol{\rho}, D, l}$, and let $\boldsymbol{\rho}$ be twice differentiable. Then $\rho$ solves the spatial Euler-Lagrange equations (13) and (14). Moreover, it fulfills the boundary conditions

$$
\begin{aligned}
-\left.\mathbf{n}^{*}\right|_{\gamma} & =b^{*} \\
-\left.\mathbf{m}^{*}\right|_{\gamma} & =\boldsymbol{\Phi}_{R}^{-*} B^{*} .
\end{aligned}
$$


Remark 2.1. Using test functions, 18 and 19 can be written equivalently as

$$
\begin{aligned}
-\left.\mathbf{n}\right|_{\gamma} \cdot \delta r & =b^{*}(\delta r) \\
-\left.\mathbf{m}\right|_{\gamma} \cdot \delta w & =B^{*}(\delta R)
\end{aligned}
$$

for all $\delta r \in T_{r} \mathbb{R}^{3}$ and $\delta R \in T_{R} \mathrm{SO}(3)$, and with $\delta w=\boldsymbol{\Phi}_{R} \delta R=\left(\delta R R^{-1}\right)_{\times}$.

Proof. Writing out $T_{\boldsymbol{\rho}} j \delta \boldsymbol{\rho}$ in 17 we obtain

$$
\begin{aligned}
0= & \int_{[0, l]} \frac{\partial W}{\partial \mathrm{v}} \cdot\left(\frac{\partial \mathrm{v}}{\partial r} \delta r+\frac{\partial \mathrm{v}}{\partial R} \delta R\right)+\frac{\partial W}{\partial \mathrm{u}} \cdot\left(\frac{\partial \mathrm{u}}{\partial r} \delta r+\frac{\partial \mathrm{u}}{\partial R} \delta R\right) d s \\
& -b^{*}\left(\left.\delta r\right|_{\gamma}\right)-B^{*}\left(\left.\delta R\right|_{\gamma}\right) .
\end{aligned}
$$

We then compute the partial derivatives of $\mathrm{v}(s)=R^{-1}(s) r^{\prime}(s)$ and $\mathrm{u}(s)=$ $\left(R(s)^{-1} R^{\prime}(s)\right)_{\times}$. For the derivatives with respect to $r$ we obtain immediately

$$
\frac{\partial \mathrm{v}}{\partial r} \delta r=R^{-1} \delta r^{\prime} \quad \text { and } \quad \frac{\partial \mathrm{u}}{\partial r} \delta r=0 .
$$

Next, we use the formula $\left(\partial\left(R^{-1}\right) / \partial R\right) \delta R=-R^{-1} \delta R R^{-1}$ to compute

$$
\frac{\partial \mathrm{v}}{\partial R} \delta R=-R^{-1}\left(\delta R R^{-1}\right) r^{\prime}
$$

Finally, using that

$$
\begin{aligned}
\frac{\partial R^{-1} R^{\prime}}{\partial R} \delta R & =-R^{-1} \delta R R^{-1} R^{\prime}+R^{-1} \delta R^{\prime} \\
& =R^{-1}\left(-\delta R R^{-1} R^{\prime} R^{-1}+\delta R^{\prime} R^{-1}\right) R=R^{-1}\left(\delta R R^{-1}\right)^{\prime} R,
\end{aligned}
$$

we obtain

$$
\begin{aligned}
\frac{\partial \mathrm{u}}{\partial R} \delta R=\frac{\partial\left(R^{-1} R^{\prime}\right)_{\times}}{\partial R} \delta R=\left(\frac{\partial R^{-1} R^{\prime}}{\partial R} \delta R\right)_{\times} & \\
& =\left(R^{-1}\left(\delta R R^{-1}\right)^{\prime} R\right)_{\times}=R^{-1}\left(\delta R R^{-1}\right)_{\times}^{\prime}
\end{aligned}
$$

Inserting these results into 20 yield

$$
\begin{aligned}
0= & \int_{[0, l]} \mathrm{n} \cdot\left(R^{-1} \delta r^{\prime}\right) d s-b^{*}\left(\left.\delta r\right|_{\gamma}\right) \\
& \left.+\int_{[0, l]}-R^{-1}\left(\delta R R^{-1}\right) r^{\prime}\right)+\mathrm{m} \cdot R^{-1}\left(\delta R R^{-1}\right)_{\times}^{\prime} d s-B^{*}\left(\left.\delta R\right|_{\gamma}\right) .
\end{aligned}
$$

Let us now define $\delta w:=\boldsymbol{\Phi}_{R} \delta R=\left(\delta R R^{-1}\right)_{\times}$, noting that $\mathbf{m}^{*}(\delta w)=B^{*}(\delta R)$. Performing integration by parts and and setting $(\mathbf{n}, \mathbf{m})=(R \mathrm{n}, R \mathrm{~m})$ as in (5) we obtain

$$
\begin{aligned}
0= & \int_{[0, l]}-(R \mathrm{n})^{\prime} \cdot \delta r d s+\left[-\left.\left(\left.\left.R\right|_{\gamma} \mathbf{n}\right|_{\gamma}\right) \cdot \delta r\right|_{\gamma}-b^{*}\left(\left.\delta r\right|_{\gamma}\right)\right] \\
& +\int_{[0, l]}-(R \mathrm{~m})^{\prime} \cdot \delta w-(R \mathrm{~m}) \cdot \delta w \times r^{\prime} d s+\left[-\left.\left(\left.\left.R\right|_{\gamma} \mathrm{m}\right|_{\gamma}\right) \cdot \delta w\right|_{\gamma}-B^{*}\left(\left.\delta R\right|_{\gamma}\right)\right] \\
= & \int_{[0, l]}-\mathbf{n}^{\prime} \cdot \delta r d s-\left[\left.\left.\mathbf{n}\right|_{\gamma} \cdot \delta r\right|_{\gamma}+b^{*}\left(\left.\delta r\right|_{\gamma}\right)\right] \\
& +\int_{[0, l]}-\mathbf{m}^{\prime} \cdot \delta w-\left(r^{\prime} \times \mathbf{m}\right) \cdot \delta w d s-\left[\left.\left.\mathbf{m}\right|_{\gamma} \cdot \delta w\right|_{\gamma}+\mathbf{\Phi}_{R}^{-*} B^{*}\left(\left.\delta w\right|_{\gamma}\right)\right] .
\end{aligned}
$$


There is no boundary term at $s=l$ by construction of the test functions. Since the equation must hold for all test functions $\delta r$ and $\delta R$, the equilibrium equations (13) and (14) follow from the integral terms. Likewise, the boundary conditions (18) and (19) follow from the non-integral terms.

Remark 2.2. The minus signs in (18) and (19) originate from the integration by parts performed in the proof, and reflect the fact that we apply Neumann conditions at the left end of the rod parameter domain. Indeed, if we use $\boldsymbol{\nu}_{r}$ to denote the unit outer normal of the rod parameter domain $[0, l]$ we get $\boldsymbol{\nu}_{r}(0)=$ -1 and $\boldsymbol{\nu}_{r}(l)=1$. A more general way to write conditions $\sqrt{18}$ and $\sqrt{19}$ would hence be

$$
\begin{aligned}
\left.\left.\mathbf{n}^{*}\right|_{\gamma} \boldsymbol{\nu}\right|_{\gamma} & =b^{*} \\
\left.\left.\mathbf{m}^{*}\right|_{\gamma} \boldsymbol{\nu}\right|_{\gamma} & =\boldsymbol{\Phi}_{R}^{-*} B^{*}
\end{aligned}
$$

which involves the outer normal boundary stresses of the rod on the left. For a simpler notation, though, we stick with the form used in Theorem 2.2 for the rest of the paper.

\section{Elastic Continua}

We now describe our model of the elastic continuum. Let $\mathbb{E}^{3}$ be a threedimensional Euclidean space, which we will use as the parameter space. A body is an open connected subset $\mathcal{B} \subset \mathbb{E}^{3}$, and a configuration of $\mathcal{B}$ is a mapping $\phi: \mathcal{B} \rightarrow \mathbb{R}^{3}$ such that $\phi(\mathcal{B})$ is open and connected, and $\phi$ has an inverse $\phi^{-1}: \phi(\mathcal{B}) \rightarrow \mathcal{B}$. We single out one configuration $\phi_{0}: \mathcal{B} \rightarrow \mathbb{R}^{3}$ and call it the reference configuration. In principle it is possible to use any configuration as the reference configuration. For simplicity we assume only that $\phi_{0}$ is a $C^{\infty}$-diffeomorphism.

We assume the boundary $\partial \mathcal{B}$ to be Lipschitz continuous and to consist of two disjoint parts $\partial_{D} \mathcal{B}$ and $\partial_{N} \mathcal{B}$ such that $\partial \mathcal{B}=\overline{\partial_{D} \mathcal{B}} \cup \overline{\partial_{N} \mathcal{B}}$. The unit boundary normals of the domain $\mathcal{B}$ are denoted by $\boldsymbol{\nu}$. Our space of admissible configurations is $\mathbf{H}^{1}(\mathcal{B})$, the usual first-order Sobolev space of three-valued functions on $\mathcal{B}$, constraint to fulfill Dirichlet conditions if present. We consider hyperelastic continua, i.e., we assume the existence of a stored energy function $\widehat{W}: \mathcal{B} \times \mathbb{R}^{3 \times 3} \rightarrow \mathbb{R}$ such that equilibrium configurations of the continuum are stationary points of the functional

$$
E(\boldsymbol{\phi})=\int_{\mathcal{B}}\left[\widehat{W}(x, \nabla \boldsymbol{\phi})-V_{f}(\boldsymbol{\phi})\right] d V-\int_{\partial_{\mathcal{N} \mathcal{B}}} V_{g}(\boldsymbol{\phi}) d A .
$$

The volume loads $V_{f}$ and surface loads $V_{g}$ are assumed to be dead loads, i.e., there are functions $f: \mathcal{B} \rightarrow \mathbb{R}^{3}$ and $g: \partial_{N} \mathcal{B} \rightarrow \mathbb{R}^{3}$ such that $V_{f}(\phi)=f \cdot \phi$ and $V_{g}(\phi)=g \cdot \phi$.

In this article we focus only on minimizers of $E$. To guarantee the existence of such minimizers, we make the following assumption.

Assumption 3.1. The energy functional $E$ is weakly lower semi-continuous and coercive in the semi-normed space $\left(\mathbf{H}^{1}(\mathcal{B}),\|\nabla \cdot\|_{\mathbf{L}_{2}(\mathcal{B})}\right)$. 
Since $\widehat{W}$ depends on $\phi$ through $\nabla \phi$ only, we cannot expect more than coercivity in the above semi-normed space. The energy $E$ will be coercive with respect to the full norm $\|\cdot\|_{\mathbf{H}^{1}}$ if $\partial_{D} \mathcal{B}$ has non-zero two-dimensional measure.

Large classes of stored energy functionals satisfy Assumption 3.1. Particular examples are linearly elastic materials, and polyconvex materials such as Mooney-Rivlin materials. In the case of linear elasticity $E$ is a quadratic energy functional. Convexity and coercivity of $E$ follow from Korn's inequality, and weak lower semi-continuity is in turn a consequence of the convexity of $E$. In the polyconvex case, showing weak lower semi-continuity of $E$ is more involved and depends on several delicate assumptions on $\widehat{W}$. Details can be found, e.g., in [8, Chap. 7].

The minimization formulation of the continuum elasticity problem is convenient, because it makes only few regularity assumptions. We will use this formulation later as the basis of our proof showing existence of solutions to coupled rod-continuum systems. However, to properly interpret the resulting dual coupling conditions, we need the weak and strong forms of the equilibrium equations as well. These can only be stated under additional smoothness assumptions.

Consider an energy minimizer $\phi_{*}$ of $E$ and assume that $E$ is Gâteauxdifferentiable at $\phi_{*}$ for all directions $\delta \phi \in \mathbf{C}^{1}(\mathcal{B})$. Then differentiation of 21] yields the corresponding Euler-Lagrange-equation

$$
\begin{aligned}
0 & =T_{\phi_{*}} E(\delta \phi) \\
& =\int_{\mathcal{B}}\left[P\left(x, \nabla \phi_{*}\right) \nabla \delta \phi-f \cdot \delta \phi\right] d V-\int_{\partial_{\mathcal{N}} \mathcal{B}} g \cdot \delta \phi d A
\end{aligned}
$$

for all test functions $\delta \phi \in \mathbf{C}^{1}(\mathcal{B})$ with a zero trace on $\partial_{D} \mathcal{B}$. Here, $P$ is the first Piola-Kirchhoff stress tensor [8, Chap. 4]

$$
P(x)=P\left(x, \nabla \phi_{*}\right)=\frac{\partial \widehat{W}\left(x, \nabla \phi_{*}\right)}{\partial \nabla \phi} .
$$

To derive a strong form of the equilibrium conditions 22 we have to integrate by parts (which is, of course, only possible if $\phi_{*}$ is sufficiently smooth), and obtain

$$
\begin{aligned}
-\operatorname{div} P=f & \text { in } \mathcal{B}, \\
P \boldsymbol{\nu}=g & \text { on } \partial_{N} \mathcal{B}
\end{aligned}
$$

in the reference domain.

\section{Coupling Conditions}

In this chapter we formulate coupling conditions for a model consisting of one Cosserat rod and one continuum, where the rod is attached by one of its endpoints to a part of the boundary of the continuum, and the other end of the rod is clamped (Figure 2). We discuss two different sets of coupling conditions, which correspond to different choices of the interface space.

Let $\mathcal{B} \subset \mathbb{E}^{3}$ as in Section 3 However, the boundary $\partial \mathcal{B}$ is now supposed to consist of two disjoint parts $\partial_{N} \mathcal{B}$ and $\Gamma$, such that $\partial \mathcal{B}=\overline{\partial_{N} \mathcal{B}} \cup \bar{\Gamma}$. We 


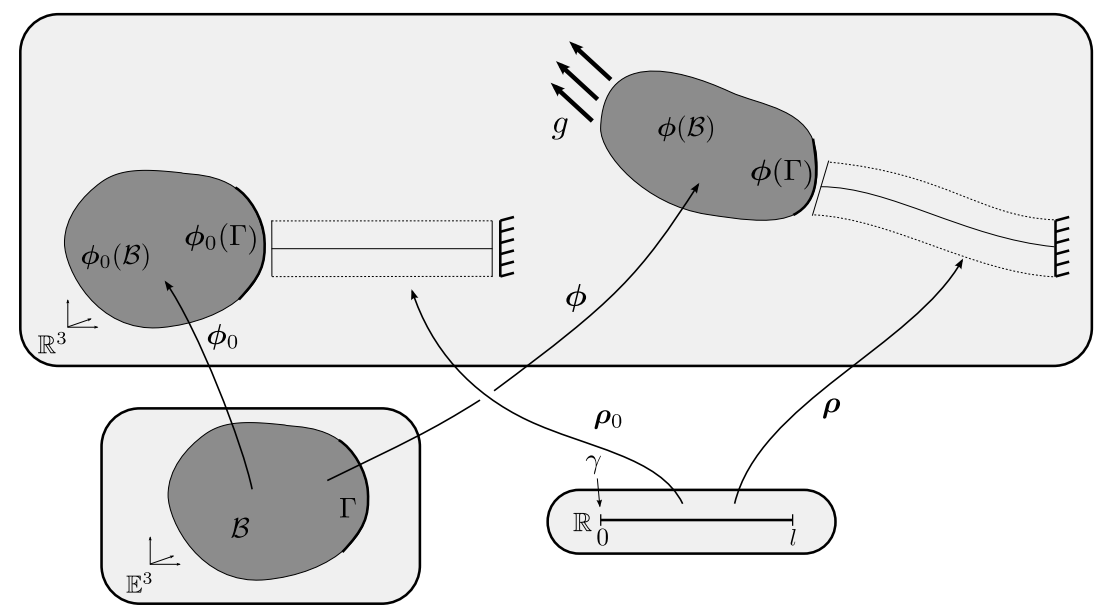

Figure 2: Coupling between a three-dimensional continuum and a Cosserat rod.

assume that $\Gamma$ has positive two-dimensional measure and contains a subset that is diffeomorphic to a disc. Additionally, we assume that the trace mapping

$$
\left.(\cdot)\right|_{\Gamma}: \mathbf{H}^{3}(\mathcal{B}) \rightarrow \mathbf{H}^{5 / 2}(\Gamma)
$$

is continuous and surjective. All these assumptions are fulfilled if $\Gamma$ is a smoothly bounded relatively open subset of $\partial \mathcal{B}$, and $\mathcal{B}$ is a $C^{2,1}$-domain (cf., e.g., [22, Sec. I $\S 8]$ ). Note that by the Sobolev embedding theorems we have the continuous embedding $\mathbf{H}^{3}(\mathcal{B}) \hookrightarrow \mathbf{C}^{1}(\mathcal{B})$, which will be needed later in view of $(22)$.

The three-dimensional object represented by $\mathcal{B}$ will couple with the rod across $\Gamma$, and we call $\Gamma$ the coupling boundary. On $\partial \mathcal{B}_{N}$ boundary forces may act. For simplicity of presentation we do not consider Dirichlet boundary conditions on any part of $\partial \mathcal{B}$, which would, however, be straightforward to incorporate.

Consider also a Cosserat rod defined on the parameter interval $[0, l]$, with smooth reference configuration $\boldsymbol{\rho}_{0}:[0, l] \rightarrow \mathrm{SE}(3)$. The boundary $\partial[0, l]$ of the parameter domain $[0, l]$ consists of the two points $\{0\}$ and $\{l\}$. We call $\gamma \subset \partial[0, l]$ the coupling boundary of the rod. To be specific, we pick $\gamma=\{0\}$. On the other boundary $s=l$ we will impose Dirichlet boundary conditions. As in Section 2 we use the constraint manifold $H_{D, l}^{1}([0, l], \mathrm{SE}(3))$ defined in 15$)$ to incorporate these Dirichlet conditions.

Our coupling conditions involve only the primal variables restricted to the coupling boundaries $\Gamma$ and $\gamma$. For the continuum model we have

$$
\left.\phi\right|_{\Gamma} \in \mathbf{H}^{1 / 2}(\Gamma),
$$

and for the rod we have

$$
\left.\boldsymbol{\rho}\right|_{\gamma}=\boldsymbol{\rho}(0)=(r(0), R(0)) \in \mathrm{SE}(3)
$$

This is the position and orientation of the rod cross-section at the coupling boundary.

To prepare for the existence results in Section 5 we will formulate the coupled problems in a variational form. First, we will write the coupling conditions as 
equations

$$
c\left(\left.\phi\right|_{\Gamma},\left.\boldsymbol{\rho}\right|_{\gamma}\right)=0
$$

with a constraint mapping

$$
c: \mathbf{H}^{1 / 2}(\Gamma) \times \mathrm{SE}(3) \rightarrow V
$$

into a linear space $V$. The precise forms of $c$ and $V$ will depend on the actual coupling conditions (see Chapters 4.1 and 4.2). Then we add (7) and (21) to obtain the total energy minimization problem

$$
\min [E(\phi)+j(\boldsymbol{\rho})]
$$

restricted to those $\boldsymbol{\phi} \in \mathbf{H}^{1}(\mathcal{B})$ and $\boldsymbol{\rho} \in H_{D, l}^{1}([0, l], \mathrm{SE}(3))$ with

$$
c\left(\left.\phi\right|_{\Gamma},\left.\boldsymbol{\rho}\right|_{\gamma}\right)=0 \text {. }
$$

The coupled problem thus assumes the form of a single constrained minimization problem, and can be treated using the calculus of variations.

In the rest of this chapter we will now discuss two alternative coupling conditions. In Section 5 we will show existence of minimizers $\left(\boldsymbol{\phi}_{*}, \boldsymbol{\rho}_{*}\right)$ to the constrained problem $(24)+\sqrt{25}$ for both of them. Then we will derive existence of Lagrange multipliers $\lambda \in V^{*}$ at a minimizer $\left(\phi_{*}, \rho_{*}\right)$ such that the first order optimality conditions are fulfilled. Finally, we will interpret this system of equations as equilibrium conditions, where the Lagrange multipliers yield the constraint forces.

\subsection{Rigid coupling}

For the first set of coupling conditions we assume that under load, the pair of coupling boundaries $\left.\phi\right|_{\Gamma}$ and $\left.\boldsymbol{\rho}\right|_{\gamma}$ moves rigidly from their reference configurations $\left.\phi_{0}\right|_{\Gamma}$ and $\left.\boldsymbol{\rho}_{0}\right|_{\gamma}$. That means there is a rigid body motion $\mathcal{R} \in \mathrm{SE}(3)$ such that both

$$
\mathcal{R}\left(\left.\phi_{0}\right|_{\Gamma}\right)=\left.\phi\right|_{\Gamma}
$$

and

$$
\mathcal{R}\left(\left.\rho_{0}\right|_{\gamma}\right)=\left.\boldsymbol{\rho}\right|_{\gamma}
$$

Here, (26) is to be understood as an action of $\mathcal{R}$ on the points $\left.\phi_{0}\right|_{\Gamma}(x)$ for almost all $x \in \Gamma$. Since both $\mathcal{R}$ and $\left.\boldsymbol{\rho}_{0}\right|_{\gamma}$ are elements of $\operatorname{SE}(3)$ we have $\mathcal{R}\left(\left.\boldsymbol{\rho}_{0}\right|_{\gamma}\right)=$ $\left.\mathcal{R} \cdot \boldsymbol{\rho}_{0}\right|_{\gamma}$, i.e., group multiplication in $\mathrm{SE}(3)$. Hence we can use the group structure of $\mathrm{SE}(3)$ to get

$$
\mathcal{R}=\left.\boldsymbol{\rho}\right|_{\gamma} \cdot\left(\left.\boldsymbol{\rho}_{0}\right|_{\gamma}\right)^{-1} .
$$

Inserting this into 26$)$ yields

$$
\left.\boldsymbol{\rho}\right|_{\gamma} \cdot\left(\left.\boldsymbol{\rho}_{0}\right|_{\gamma}\right)^{-1}\left(\left.\phi_{0}\right|_{\Gamma}\right)=\left.\phi\right|_{\Gamma}
$$

Introduce $r, r_{0} \in \mathbb{R}^{3}$ and $R, R_{0} \in \mathrm{SO}(3)$ such that $\left.\boldsymbol{\rho}\right|_{\gamma}=(r, R)$ and $\left.\boldsymbol{\rho}_{0}\right|_{\gamma}=$ $\left(r_{0}, R_{0}\right)$. The multiplication rule (1) in $\mathrm{SE}(3)$ gives

$$
\left.\boldsymbol{\rho}\right|_{\gamma} \cdot\left(\left.\boldsymbol{\rho}_{0}\right|_{\gamma}\right)^{-1}=(r, R) \cdot\left(-R_{0}^{-1} r_{0}, R_{0}^{-1}\right)=\left(-R R_{0}^{-1} r_{0}+r, R R_{0}^{-1}\right) .
$$

Hence we obtain the coupling condition

$$
\left(-R R_{0}^{-1} r_{0}+r, R R_{0}^{-1}\right)\left(\left.\phi_{0}\right|_{\Gamma}\right)=\left.\phi\right|_{\Gamma}
$$


or, equivalently,

$$
R R_{0}^{-1}\left(\left.\phi_{0}\right|_{\Gamma}\right)-R R_{0}^{-1} r_{0}+r=\left.\phi\right|_{\Gamma} .
$$

Note that this is an equation in the function space $\mathbf{H}^{1 / 2}(\Gamma)$, i.e., it holds pointwise almost everywhere on $\Gamma$.

We now write this condition in the variational form of the previous section. For this we set $V=\mathbf{H}^{1 / 2}(\Gamma)$ and define the constraint function

$$
\begin{aligned}
c_{\mathrm{r}} & : \mathbf{H}^{1 / 2}(\Gamma) \times \mathrm{SE}(3) \rightarrow \mathbf{H}^{1 / 2}(\Gamma) \\
c_{\mathrm{r}}\left(\left.\boldsymbol{\phi}\right|_{\Gamma},\left.\boldsymbol{\rho}\right|_{\gamma}\right) & =\left(\left.\phi\right|_{\Gamma}-r\right)-R R_{0}^{-1}\left(\left.\phi_{0}\right|_{\Gamma}-r_{0}\right) .
\end{aligned}
$$

It is evident that $c_{\mathrm{r}}\left(\left.\boldsymbol{\phi}\right|_{\Gamma},\left.\boldsymbol{\rho}\right|_{\gamma}\right)=0$ if and only if $(29)$ holds.

The following two lemmas state a few technical properties of $c_{\mathrm{r}}$.

Lemma 4.1. The subset of $\mathbf{H}^{1}(\mathcal{B}) \times H_{D, l}^{1}([0, l], \operatorname{SE}(3))$ defined by $c_{r}\left(\left.\boldsymbol{\phi}\right|_{\Gamma},\left.\boldsymbol{\rho}\right|_{\gamma}\right)=$ 0 is weakly closed.

Proof. Consider a weakly converging sequence $\left(\phi_{k}, \boldsymbol{\rho}_{k}\right) \rightarrow\left(\phi_{*}, \boldsymbol{\rho}_{*}\right)$ such that $c_{\mathrm{r}}\left(\left.\phi_{k}\right|_{\Gamma},\left.\boldsymbol{\rho}_{k}\right|_{\gamma}\right)=0$ for all $k \in \mathbb{N}$. Since the trace operators are linear and continuous in the spaces that we have chosen, the sequence $\left(v_{k},\left(r_{k}, R_{k}\right)\right):=$ $\left(\left.\boldsymbol{\phi}_{k}\right|_{\Gamma},\left.\boldsymbol{\rho}_{k}\right|_{\gamma}\right)$ is weakly converging as well, with limit $\left(v_{*},\left(r_{*}, R_{*}\right)\right)=\left(\left.\boldsymbol{\phi}_{*}\right|_{\Gamma},\left.\boldsymbol{\rho}_{*}\right|_{\gamma}\right)$. We have to show that $c_{\mathrm{r}}\left(v_{*},\left(r_{*}, R_{*}\right)\right)=0$. Since $\mathrm{SE}(3)$ is finite dimensional we conclude that $\left(r_{k}, R_{k}\right) \rightarrow\left(r_{*}, R_{*}\right)$ strongly. It follows that

$$
\left(r_{k}+R_{k} R_{0}^{-1}\left(\left.\phi_{0}\right|_{\Gamma}-r_{0}\right)\right) \rightarrow\left(r_{*}+R_{*} R_{0}^{-1}\left(\left.\phi_{0}\right|_{\Gamma}-r_{0}\right)\right)
$$

in $\mathbf{H}^{1 / 2}(\Gamma)$. Hence in the equation

$$
0=c_{\mathrm{r}}\left(v_{k},\left(r_{k}, R_{k}\right)\right)=\left(v_{k}-r_{k}-R_{k} R_{0}^{-1}\left(\left.\phi_{0}\right|_{\Gamma}-r_{0}\right)\right),
$$

the right hand side converges weakly to

$$
\left(v_{*}-r_{*}-R_{*} R_{0}^{-1}\left(\left.\phi_{0}\right|_{\Gamma}-r_{0}\right)\right)=c_{\mathrm{r}}\left(v_{*},\left(r_{*}, R_{*}\right)\right) .
$$

Thus $c_{\mathrm{r}}\left(v_{*},\left(r_{*}, R_{*}\right)\right)=0$.

Lemma 4.2. The derivative $T$ of $c_{r}$ at $\left(\left.\phi\right|_{\Gamma},\left.\boldsymbol{\rho}\right|_{\gamma}\right)=\left(\left.\phi\right|_{\Gamma},(r, R)\right)$ is a surjective mapping

$$
T_{\left(\left.\boldsymbol{\phi}\right|_{\Gamma},\left.\boldsymbol{\rho}\right|_{\gamma}\right)} c_{r}: \mathbf{H}^{1 / 2}(\Gamma) \times T_{\left.\boldsymbol{\rho}\right|_{\gamma}} \mathrm{SE}(3) \rightarrow \mathbf{H}^{1 / 2}(\Gamma)
$$

and is given by

$$
T_{\left(\left.\boldsymbol{\phi}\right|_{\Gamma},\left.\boldsymbol{\rho}\right|_{\gamma}\right)} c_{r}\left(\left.\delta \boldsymbol{\phi}\right|_{\Gamma},(\delta r, \delta R)\right)=\left(\left.\delta \phi\right|_{\Gamma}-\delta r\right)-\delta R R_{0}^{-1}\left(\left.\phi_{0}\right|_{\Gamma}-r_{0}\right) .
$$

Proof. The formula for the derivative follows from the linearity of $c_{\mathrm{r}}$ in $\phi, r$, and $R$. Setting $(\delta r, \delta R)=(0,0)$ we immediately obtain surjectivity of $T_{\left(\left.\boldsymbol{\phi}\right|_{\Gamma},\left.\boldsymbol{\rho}\right|_{\gamma}\right)} c_{\mathrm{r}}$, since

$$
T_{\left(\left.\boldsymbol{\phi}\right|_{\Gamma},\left.\boldsymbol{\rho}\right|_{\gamma}\right)} c_{\mathrm{r}}\left(\left.\delta \phi\right|_{\Gamma},(0,0)\right)=\left.\delta \phi\right|_{\Gamma} .
$$

Note that in Lemma 4.2 we have used the canonical identifications of the linear space $\mathbf{H}^{1 / 2}(\Gamma)$ with its tangent spaces. 
Remark 4.1. Clearly, $T_{\left(\left.\boldsymbol{\phi}\right|_{\Gamma},\left.\boldsymbol{\rho}\right|_{\gamma}\right)} c_{r}$ remains surjective if we replace the domain and codomain space $\mathbf{H}^{1 / 2}(\Gamma)$ by any subspace.

Finally, we show a density result, which will be needed in Section 6. Let us define the subspace

$$
K_{\phi, \boldsymbol{\rho}}^{\mathrm{r}}:=\left\{(\delta \boldsymbol{\phi}, \delta \boldsymbol{\rho}) \in \mathbf{H}^{1}(\mathcal{B}) \times Y_{\boldsymbol{\rho}, D, l} \mid T_{\left(\left.\boldsymbol{\phi}\right|_{\Gamma},\left.\boldsymbol{\rho}\right|_{\gamma}\right)} c_{\mathrm{r}}\left(\left.\delta \phi\right|_{\Gamma},\left.\delta \boldsymbol{\rho}\right|_{\gamma}\right)=0\right\} .
$$

Proposition 4.1. The intersection

$$
K_{\boldsymbol{\phi}, \boldsymbol{\rho}}^{r} \cap \mathbf{H}^{3}(\mathcal{B}) \times H^{2}\left([0, l], \mathbb{R}^{3} \times \mathbb{R}^{3 \times 3}\right)
$$

is dense in $K_{\phi, \rho}^{r}$.

Proof. We will show that any given $(\delta \phi, \delta \boldsymbol{\rho}) \in K_{\phi, \rho}$ can be approximated by an element of $K_{\phi, \rho}^{\mathrm{r}} \cap \mathbf{H}^{3}(\mathcal{B}) \times H^{2}\left([0, l], \mathbb{R}^{3} \times \mathbb{R}^{3 \times 3}\right)$. By the well known density result in Sobolev spaces $\delta \boldsymbol{\rho}$ can be approximated by $C^{\infty}$ functions, and thus also by a $H^{2}$ function $\tilde{\boldsymbol{\rho}}$; we may choose $\tilde{\boldsymbol{\rho}}$ such that $\left.\tilde{\boldsymbol{\rho}}\right|_{\gamma}=\left.\delta \boldsymbol{\rho}\right|_{\gamma}$. Since $\left.\delta \boldsymbol{\rho}\right|_{\gamma}$ acts as an affine mapping on $\Gamma,\left(\left.\delta \phi\right|_{\Gamma},\left.\delta \boldsymbol{\rho}\right|_{\gamma}\right) \in \operatorname{ker} T_{\left(\left.\boldsymbol{\phi}\right|_{\Gamma},\left.\boldsymbol{\rho}\right|_{\gamma}\right)} c_{\mathrm{r}}$ implies that $\left.\delta \boldsymbol{\phi}\right|_{\Gamma}$ is an affine mapping as well, and thus in particular an element of $\mathbf{H}^{5 / 2}(\Gamma)$. By surjectivity of the trace operator (23) we find an extension $\phi_{T} \in \mathbf{H}^{3}(\mathcal{B})$ of $\left.\delta \phi\right|_{\Gamma}$ such that $\left.\phi_{T}\right|_{\Gamma}=\left.\delta \phi\right|_{\Gamma}$. By density in Sobolev spaces, we may now approximate $\delta \phi-\phi_{T}$ (which satisfies $\left.\left(\delta \phi-\phi_{T}\right)\right|_{\Gamma}=0$ ) by some $\phi_{A} \in \mathbf{H}^{3}(\mathcal{B})$, such that $\left.\phi_{A}\right|_{\Gamma}=0$. Then $\phi_{T}+\phi_{A} \in \mathbf{H}^{3}(\mathcal{B})$ satisfies $\left.\left(\phi_{T}+\phi_{A}\right)\right|_{\Gamma}=\left.\delta \phi\right|_{\Gamma}$ and approximates $\delta \phi$ in $\mathbf{H}^{1}(\mathcal{B})$. Hence, the pair $\left(\boldsymbol{\phi}_{T}+\boldsymbol{\phi}_{A}, \tilde{\boldsymbol{\rho}}\right)$ is in $K_{\boldsymbol{\phi}, \boldsymbol{\rho}} \cap \mathbf{H}^{3}(\mathcal{B}) \times H^{2}\left([0, l], \mathbb{R}^{3} \times \mathbb{R}^{3 \times 3}\right)$, and approximates $(\delta \boldsymbol{\phi}, \delta \boldsymbol{\rho})$.

\subsection{Averaged Coupling}

Our second coupling condition is formulated in $\mathfrak{s e}(3)$, the Lie algebra of the rod configuration space restricted to $\gamma$. The main idea here is to drop the condition (26) that $\left.\phi\right|_{\Gamma}$ is transformed by a rigid body motion. Instead, for any $\left.\phi\right|_{\Gamma}$ we seek a rigid body motion $\mathcal{R}$ that minimizes the least squares error between $\left.\phi\right|_{\Gamma}$ and $\mathcal{R}\left(\left.\phi_{0}\right|_{\Gamma}\right)$. Thus, (26) is replaced by

$$
\mathcal{R} \in \underset{\mathcal{Q} \in \operatorname{SE}(3)}{\arg \min } \frac{1}{2}\left\|\left.\phi\right|_{\Gamma}-\mathcal{Q}\left(\left.\phi_{0}\right|_{\Gamma}\right)\right\|_{\mathbf{L}_{2}(\Gamma)}^{2},
$$

which, together with 27) yields our second set of coupling conditions.

An alternative formulation gets rid of the minimization problem. Note that instead of writing the orientation condition on $\mathfrak{s o}(3)$, we choose a formulation in $\mathbb{R}^{3}$ using axial vectors. This allows an easier interpretation.

Proposition 4.2. Assume that $\left(\left.\phi\right|_{\Gamma},\left.\boldsymbol{\rho}\right|_{\gamma}\right)$ are such that (27) and (32) hold, and write $\left.\boldsymbol{\rho}\right|_{\gamma}$ as $(r, R)$. Then we also have the coupling conditions

$$
\begin{aligned}
& 0=\int_{\Gamma}\left(\left.\phi\right|_{\Gamma}-r\right)-R R_{0}^{-1}\left(\left.\phi_{0}\right|_{\Gamma}-r_{0}\right) d A \\
& 0=\int_{\Gamma}\left(\left.\phi\right|_{\Gamma}-r\right) \times R R_{0}^{-1}\left(\left.\phi_{0}\right|_{\Gamma}-r_{0}\right) d A .
\end{aligned}
$$


Proof. In the following we will frequently use the function

$$
\psi: \Gamma \rightarrow \mathbb{R}^{3}, \quad \psi(x):=R_{0}^{-1}\left(\left.\phi_{0}\right|_{\Gamma}(x)-r_{0}\right)
$$

to shorten the notation. Let $\mathcal{R}$ be a minimizer of (32). Then it satisfies the first order optimality conditions

$$
0=\left\langle\left.\phi\right|_{\Gamma}-\mathcal{R}\left(\left.\phi_{0}\right|_{\Gamma}\right),-\delta \mathcal{R}\left(\left.\phi_{0}\right|_{\Gamma}\right)\right\rangle_{\mathbf{L}_{2}(\Gamma)} \quad \forall \delta \mathcal{R} \in T_{\mathcal{R}} \mathrm{SE}(3) .
$$

Using the coupling condition (27), which implies (28), we compute

$$
\begin{aligned}
\mathcal{R}\left(\left.\phi_{0}\right|_{\Gamma}\right) & =r+R R_{0}^{-1}\left(\left.\phi_{0}\right|_{\Gamma}-r_{0}\right)=r+R \psi \\
\delta \mathcal{R}\left(\left.\phi_{0}\right|_{\Gamma}\right) & =\delta r+\delta R R_{0}^{-1}\left(\left.\phi_{0}\right|_{\Gamma}-r_{0}\right)=\delta r+\delta R \psi,
\end{aligned}
$$

(with $(\delta r, \delta R) \in T_{\left.\boldsymbol{\rho}\right|_{\gamma}} \mathrm{SE}(3)$ ). Inserting this into (36) we obtain

$$
0=\left\langle\left.\phi\right|_{\Gamma}-r-R \psi,-\delta r-\delta R \psi\right\rangle_{\mathbf{L}_{2}(\Gamma)} \quad \forall \delta r \in \mathbb{R}^{3}, \delta R \in T_{R} \mathrm{SO}(3) .
$$

Hence, setting $\delta R=0$ yields

$$
0=\left\langle\left.\phi\right|_{\Gamma}-r-R \psi, \delta r\right\rangle_{\mathbf{L}_{2}(\Gamma)}=\int_{\Gamma}\left(\left.\phi\right|_{\Gamma}-r-R \psi\right) d A \cdot \delta r \quad \forall \delta r \in \mathbb{R}^{3},
$$

which implies (33).

If we set $\delta r=0$ instead, we obtain

$$
0=\left\langle\left.\phi\right|_{\Gamma}-r-R \psi, \delta R \psi\right\rangle_{\mathbf{L}_{2}(\Gamma)} \quad \forall \delta R \in T_{R} \mathrm{SO}(3) .
$$

Using the spatial representation (2) we may write $\delta R=\mathbf{U} R$ for some $\mathbf{U} \in \mathfrak{s o}(3)$, which implies

$$
0=\left\langle\left.\phi\right|_{\Gamma}-r, \mathbf{U} R \psi\right\rangle_{\mathbf{L}_{2}(\Gamma)} \quad \forall \mathbf{U} \in \mathfrak{s o}(3),
$$

because in (37) we have

$$
\langle R \psi, \delta R \psi\rangle=\langle R \psi, \mathbf{U} R \psi\rangle=0
$$

by skew-symmetry of $\mathbf{U}$. Defining $\mathbf{u}:=\mathbf{U}_{\times}$we can continue

$$
\begin{aligned}
0 & =\left\langle\left.\phi\right|_{\Gamma}-r, \mathbf{u} \times R \psi\right\rangle_{\mathbf{L}_{2}(\Gamma)}=\int_{\Gamma}\left(\left.\phi\right|_{\Gamma}-r\right) \cdot(\mathbf{u} \times R \psi) d A \\
& =\int_{\Gamma} \mathbf{u} \cdot\left(R \psi \times\left(\left.\phi\right|_{\Gamma}-r\right)\right) d A=\int_{\Gamma} R \psi \times\left(\left.\phi\right|_{\Gamma}-r\right) d A \cdot \mathbf{u} \quad \text { for all } \mathbf{u} \in \mathbb{R}^{3},
\end{aligned}
$$

where we have used (4). This implies (34).

Following our variational program we rewrite (33) and (34) as a constraint function. This time, the function is defined as

$$
\begin{gathered}
c_{\mathrm{a}}: \mathbf{H}^{1 / 2}(\Gamma) \times \operatorname{SE}(3) \rightarrow \mathbb{R}^{3} \times \mathbb{R}^{3} \\
c_{\mathrm{a}}\left(\left.\phi\right|_{\Gamma},\left.\boldsymbol{\rho}\right|_{\gamma}\right)=\left(\begin{array}{l}
\int_{\Gamma}\left(\left.\phi\right|_{\Gamma}-r\right)-R R_{0}^{-1}\left(\left.\phi_{0}\right|_{\Gamma}-r_{0}\right) d A \\
\int_{\Gamma}\left(\left.\phi\right|_{\Gamma}-r\right) \times R R_{0}^{-1}\left(\left.\phi_{0}\right|_{\Gamma}-r_{0}\right) d A
\end{array}\right) .
\end{gathered}
$$

We prove the same properties for $c_{\mathrm{a}}$ as for $c_{\mathrm{r}}$. 
Lemma 4.3. The subset of $\mathbf{H}^{1}(\mathcal{B}) \times H_{D, l}^{1}([0, l], \operatorname{SE}(3))$ defined by $c_{\mathrm{a}}\left(\left.\boldsymbol{\phi}\right|_{\Gamma},\left.\boldsymbol{\rho}\right|_{\gamma}\right)=$ 0 is weakly closed.

Proof. As in the proof of Lemma 4.1 for rigid coupling, this follows from linearity of $c_{\mathrm{a}}$ with respect to $\phi$, and continuity of $c_{\mathrm{a}}$ with respect to variables $(r, R)$ in the finite dimensional space $\mathrm{SE}(3)$.

Lemma 4.4. The derivative of $c_{a}$ is a surjective mapping

$$
T_{\left(\left.\boldsymbol{\phi}\right|_{\Gamma},\left.\boldsymbol{\rho}\right|_{\gamma}\right)} c_{a}: \mathbf{H}^{1 / 2}(\Gamma) \times T_{\left.\boldsymbol{\rho}\right|_{\gamma}} \mathrm{SE}(3) \rightarrow \mathbb{R}^{3} \times \mathbb{R}^{3},
$$

and is given by

$$
T_{\left(\left.\boldsymbol{\phi}\right|_{\Gamma},\left.\boldsymbol{\rho}\right|_{\gamma}\right)} c_{a}\left(\left.\delta \phi\right|_{\Gamma},(\delta r, \delta R)\right)=\left(\begin{array}{l}
\int_{\Gamma}\left(\left.\delta \phi\right|_{\Gamma}-\delta r\right)-\delta R R_{0}^{-1}\left(\left.\phi_{0}\right|_{\Gamma}-r_{0}\right) d A \\
\int_{\Gamma}\left(\left.\delta \phi\right|_{\Gamma}-\delta r\right) \times R R_{0}^{-1}\left(\left.\phi_{0}\right|_{\Gamma}-r_{0}\right) d A \\
+\int_{\Gamma}\left(\left.\phi\right|_{\Gamma}-r\right) \times \delta R R_{0}^{-1}\left(\left.\phi_{0}\right|_{\Gamma}-r_{0}\right) d A
\end{array}\right) .
$$

Proof. Equation (39) follows from the product rule for the cross product. To prove surjectivity of $T_{\left(\left.\boldsymbol{\phi}\right|_{\Gamma},\left.\boldsymbol{\rho}\right|_{\gamma}\right)} c_{\mathrm{a}}$, let $\left(w_{1}, w_{2}\right) \in \mathbb{R}^{3} \times \mathbb{R}^{3}$. We will show that

$$
\left\langle T_{\left(\left.\boldsymbol{\phi}\right|_{\Gamma},\left.\boldsymbol{\rho}\right|_{\gamma}\right)} c_{\mathrm{a}}\left(\left.\delta \boldsymbol{\phi}\right|_{\Gamma},(\delta r, \delta R)\right),\left(w_{1}, w_{2}\right)\right\rangle_{\mathbb{R}^{3} \times \mathbb{R}^{3}}=\left.0 \quad \forall \delta \phi\right|_{\Gamma}, \delta r, \delta R
$$

implies $\left(w_{1}, w_{2}\right)=(0,0)$.

Set $\delta r=0, \delta R=0$ in 40 to obtain

$$
\left.\int_{\Gamma} \delta \boldsymbol{\phi}\right|_{\Gamma}(x) \cdot w_{1}+\left(\left.\delta \boldsymbol{\phi}\right|_{\Gamma}(x) \times R \psi(x)\right) \cdot w_{2} d A=0,
$$

where we use again the function $\psi$ defined in (35). By (4), this is the same as

$$
\left.\int_{\Gamma} \delta \boldsymbol{\phi}\right|_{\Gamma}(x) \cdot\left(w_{1}-w_{2} \times R \psi(x)\right) d A=0 .
$$

Since this holds for all $\left.\delta \phi\right|_{\Gamma} \in \mathbf{H}^{1 / 2}(\Gamma)$ it follows that

$$
w_{1}-w_{2} \times R \psi(x)=0 \quad \forall x \in \Gamma .
$$

Since $\phi_{0}$ is a diffeomorphism, so is $R \psi=R R_{0}^{-1}\left(\left.\phi_{0}\right|_{\Gamma}-r_{0}\right)$, the range $I=$ $R \psi(\Gamma)$ of which thus contains a subset that is diffeomorphic to a disc by our assumptions on $\Gamma$. Hence, we can find three points $p_{1}, p_{2}, p_{3}$ in $I$ which are not collinear. Then (41) yields $w_{2} \times p_{1}=w_{2} \times p_{2}=w_{2} \times p_{3}=w_{1}$, and hence $w_{2} \times\left(p_{1}-p_{2}\right)=w_{2} \times\left(p_{1}-p_{3}\right)=0$. It follows that any nonzero $w_{2}$ would be parallel to both $p_{1}-p_{2}$ and $p_{1}-p_{3}$. But since these are linearly independent, we conclude $w_{2}=0$ and thus also $w_{1}=0$.

Remark 4.2. As one can see from the proof, the same surjectivity result holds, if in the domain of $T_{\left(\left.\boldsymbol{\phi}\right|_{\Gamma},\left.\boldsymbol{\rho}\right|_{\gamma}\right)} c_{a}$ the space $\mathbf{H}^{1 / 2}(\Gamma)$ is replaced by another space that contains $\mathbf{C}^{\infty}(\Gamma)$.

Finally, we need a density result, which will be a consequence of the following general lemma. 
Lemma 4.5. Let $X$ and $\tilde{X}$ be two Banach spaces, such that $\tilde{X}$ is densely and continuously embedded into $X$. Let $Y$ be a Banach space and $A: X \rightarrow Y$ continuous and surjective. Assume that the restriction $\tilde{A}: \tilde{X} \rightarrow Y$ of $A$ is also surjective. Then $\operatorname{ker} \tilde{A}$ is dense in $\operatorname{ker} A$.

Proof. Let $x \in \operatorname{ker} A$ be given. We will construct a sequence in $\operatorname{ker} \tilde{A}$ that approximates $x$ in $X$. To this end, consider a sequence $x_{k}$ in $\tilde{X}$ with $x_{k} \rightarrow x$ in $X$. By continuity of $A$ it follows that $y_{k}:=A x_{k}=\tilde{A} x_{k} \rightarrow A x=0$ in $Y$. Hence, since $\tilde{A}$ is surjective, and thus an open mapping, there is a sequence $v_{k} \rightarrow 0$ in $\tilde{X}$, such that $y_{k}=\tilde{A} v_{k}$. Consequently, $w_{k}:=x_{k}-v_{k} \in \operatorname{ker} \tilde{A}$. Since $\left\|x-w_{k}\right\|_{X} \leq\left\|x-x_{k}\right\|_{X}+\left\|v_{k}\right\|_{X} \leq\left\|x-x_{k}\right\|_{X}+c\left\|v_{k}\right\|_{\tilde{X}} \rightarrow 0$, we have shown existence of a sequence $w_{k}$ in ker $\tilde{A}$ that converges to $x$ in $X$. This gives our assertion.

In analogy to the last section let us now define the subspace

$$
K_{\boldsymbol{\phi}, \boldsymbol{\rho}}^{\mathrm{a}}:=\left\{(\delta \boldsymbol{\phi}, \delta \boldsymbol{\rho}) \in \mathbf{H}^{1}(\mathcal{B}) \times Y_{\boldsymbol{\rho}, D, l} \mid T_{\left(\left.\boldsymbol{\phi}\right|_{\Gamma},\left.\boldsymbol{\rho}\right|_{\gamma}\right)} c_{\mathrm{a}}\left(\left.\delta \boldsymbol{\phi}\right|_{\Gamma},\left.\delta \boldsymbol{\rho}\right|_{\gamma}\right)=0\right\} .
$$

Then Remark 4.2 and Lemma 4.5 imply the following:

Corollary 4.1. The intersection

$$
K_{\phi, \rho}^{a} \cap \mathbf{H}^{3}(\mathcal{B}) \times H^{2}\left([0, l], \mathbb{R}^{3} \times \mathbb{R}^{3 \times 3}\right)
$$

is dense in $K_{\phi, \rho}^{a}$.

Proof. Defining $A$ as the composition of $T_{\left(\left.\phi\right|_{\Gamma},\left.\boldsymbol{\rho}\right|_{\gamma}\right)} c_{\mathrm{a}}$ and the trace operators $\left.\right|_{\Gamma}$ and $\left.\right|_{\gamma}$ (i.e. $\left.A(\delta \boldsymbol{\phi}, \delta \boldsymbol{\rho}):=T_{\left(\left.\boldsymbol{\phi}\right|_{\Gamma},\left.\boldsymbol{\rho}\right|_{\gamma}\right)} c_{\mathrm{a}}\left(\left.\delta \boldsymbol{\phi}\right|_{\Gamma},\left.\delta \boldsymbol{\rho}\right|_{\gamma}\right)\right)$, it is easy to see that $A$ is continuous, linear, and surjective due to Lemma 4.4 and the surjectivity and continuity of the trace operators. Moreover, by definition $K_{\phi, \rho}^{\mathrm{a}}=\operatorname{ker} A$. If we define $\tilde{A}$ as the restriction of $A$ to $\mathbf{H}^{3}(\mathcal{B}) \times H^{2}\left([0, l], \mathbb{R}^{3} \times \mathbb{R}^{3 \times 3}\right)$, we can conclude continuity and surjectivity of $\tilde{A}$ as well, taking into account (23) and Remark 4.2 . Hence, Lemma 4.5 applies and yields the desired result.

\subsection{Further coupling conditions}

The heterogeneous structure of our coupling problem, involving rotation degrees of freedom on only one side, lends itself to propose even more coupling conditions than the two discussed in the previous section. In this sense our setting differs from the linear one treated in 4, where two different conditions very similar to ours appeared as canonical. We will here briefly discuss two more coupling conditions.

The first author proposed the following conditions in [19. First, with $\left.\boldsymbol{\rho}\right|_{\gamma}=$ $(r, R)$ we require that the centerline at the coupling boundary $\gamma$ be at the center of mass of the continuum coupling boundary

$$
\frac{1}{|\Gamma|} \int_{\Gamma} \phi(x) d A=r
$$

We then define an average orientation of the continuum configuration at $\Gamma$. Using the deformation gradient $\boldsymbol{\nabla} \phi(x)$ we first define the average deformation of the interface boundary $\Gamma$ as

$$
\mathcal{F}_{\Gamma}(\phi)=\frac{1}{|\Gamma|} \int_{\Gamma} \nabla \phi(x) d A .
$$


For sufficiently well-behaved $\phi$ the matrix $\mathcal{F}_{\Gamma}(\phi)$ can then be split up uniquely into a rotation and a stretching, using the polar decomposition. Let polar $\left(\mathcal{F}_{\Gamma}(\phi)\right)$ be the rotation part of the polar decomposition of $\mathcal{F}_{\Gamma}(\phi)$. We call polar $\left(\mathcal{F}_{\Gamma}(\phi)\right)$ the average orientation of $\Gamma$ induced by $\phi$.

The average orientation can now be set in relation to $R$, the orientation of the cross-section at the rod coupling boundary $\gamma$. We postulate the condition

$$
\operatorname{polar}\left(\mathcal{F}_{\Gamma}(\phi)\right) R_{0}=R,
$$

where $R_{0} \in \mathrm{SO}(3)$ is the rotational part of $\left.\boldsymbol{\rho}_{0}\right|_{\gamma}$. The condition is constructed such that it is fulfilled in particular by the reference configurations $\phi_{0}, \rho_{0}$, provided that $\phi_{0}=\mathrm{Id}$.

Equation (45) is an equation in the three-dimensional space $\mathrm{SO}(3)$. Together with (43) we get six independent conditions for the six primal rod variables. Existence of solutions for these conditions was shown in [18] using a fixed-point argument, but only under very restrictive symmetry assumptions.

The coupling conditions (43) and (45) appear to be related to the notion of averaged coupling introduced in Chapter 4.2. Indeed, (33) reduces to (43) under the assumption that $r_{0}=|\Gamma|^{-1} \int_{\Gamma} \phi_{0} d A$. Formally, Equation 45 is close to (27), however the precise relationship between (34) and (45) is unclear. We suspect (45) not to be a special case of (34).

The conditions (43) and (45) are dissatisfactory for several reasons. First of all they are challenging to handle analytically, because of the implicit definition of the polar decomposition. Secondly, they implicitly require additional regularity assumptions. Indeed, we take the continuum displacement field $\phi$ to be in $\mathbf{H}^{1}(\Omega)$. The usual Sobolev trace theorems then give us a trace of only the normal component of $\nabla \phi$ only in $\mathbf{H}^{-1 / 2}(\Gamma)$ 21, Thm.1.2]. However, for the integral in (44) to make sense we would need the complete trace to exist and to be in $\mathbf{L}_{1}$. This is a severe functional analytic obstacle for a rigorous treatment of such kinds of conditions. Finally, the averaging (44) only leads to a regular matrix if $\phi$ is sufficiently well-defined. This may not be the case in finite-strain mechanics.

This last problem is much less severe if the averaging is done in $\mathrm{SO}(3)$ instead of $\mathbb{R}^{3 \times 3}$. This leads to a set of conditions that are much more elegant, and provide well-defined averages on a much larger set of configurations. Averaging in $\mathrm{SO}(3)$ is possible using the Riemannian center of mass. One obtains the following definition for an average orientation of $\Gamma$ under $\phi$

$$
O_{\Gamma}(\phi):=\underset{Q \in \operatorname{SO}(3)}{\arg \min } \frac{1}{2} \int_{\Gamma} \operatorname{dist}(\operatorname{polar}(\nabla \phi(x)), Q)^{2} d A,
$$

where $\operatorname{dist}(\cdot, \cdot)$ is the Riemannian distance in $\mathrm{SO}(3)$. Well-posedness of the minimization problem is given in a large and fairly well-defined set of configurations [13. With this definition we can state a new coupling condition for the orientation

$$
O_{\Gamma}(\phi) R_{0}=R,
$$

which is identical to (45) except for the definition of average orientation.

It is even possible to include the translational coupling condition 43 and obtain a single condition

$$
\left(r, R R_{0}^{-1}\right)=\underset{(q, Q) \in \operatorname{SE}(3)}{\arg \min } \frac{1}{2} \int_{\Gamma} \operatorname{dist}((\phi(x), \operatorname{polar}(\nabla \phi)),(q, Q))^{2} d A,
$$


where we have used that $|\Gamma|^{-1} \int_{\Gamma} \phi d A=\arg \min _{q \in \mathbb{R}^{3}} \int_{\Gamma}\|q-\phi(x)\|^{2} d A$.

The approach based on the Riemannian center of mass is much more concise and mathematically elegant than the one based on (44). However, it suffers from the same regularity problems. Also, the definition of the average as a minimizer in a nonlinear space makes the definition even more difficult to grasp analytically. We will not treat those conditions any further in this article.

\section{Existence of energy minimizers for the cou- pled problem}

This chapter is devoted to showing the existence of energy minimizers for the minimization problem (24) with (25). These minimizers are solutions of the coupled problem.

To proceed, we denote by $\overline{\mathbb{R}}:=\mathbb{R} \cup\{+\infty\}$ the set of extended real values, and recall the well-known definition of the indicator functional $\iota_{S}: X \rightarrow \overline{\mathbb{R}}$ of a set $S \subset X$

$$
\iota_{S}(x):= \begin{cases}0 & x \in S, \\ \infty & x \notin S .\end{cases}
$$

Finally we define the feasible set

$$
F:=\left\{(\boldsymbol{\phi}, \boldsymbol{\rho}) \in \mathbf{H}^{1}(\mathcal{B}) \times H_{D, l}^{1}([0, l], \operatorname{SE}(3)) \mid c\left(\left.\phi\right|_{\Gamma},\left.\boldsymbol{\rho}\right|_{\gamma}\right)=0\right\},
$$

where $c$ is a placeholder for either $c_{\mathrm{r}}(30)$ or $c_{\mathrm{a}}(38)$.

Lemma 5.1. Any bounded sequence in $\mathbf{H}^{1}(\mathcal{B}) \times H_{D, l}^{1}([0, l], \mathrm{SE}(3))$ has a weakly converging subsequence with limit in $\mathbf{H}^{1}(\mathcal{B}) \times H_{D, l}^{1}([0, l], \mathrm{SE}(3))$. The functional $\iota_{F}: \mathbf{H}^{1}(\mathcal{B}) \times H_{D, l}^{1}([0, l], \mathrm{SE}(3)) \rightarrow \overline{\mathbb{R}}$ is weakly lower semi-continuous.

Proof. Since $\mathbf{H}^{1}(\mathcal{B})$ is a Hilbert space, and hence reflexive, any bounded sequence in $\mathbf{H}^{1}(\mathcal{B})$ has a weakly converging subsequence. In contrast (see Section 2 $H_{D, l}^{1}([0, l], \mathrm{SE}(3))$ is an infinite dimensional nonlinear manifold, embedded in the Sobolev space $H^{1}\left([0, l], \mathbb{R}^{3} \times \mathbb{R}^{3 \times 3}\right)$. Thus, a bounded sequence $\boldsymbol{\rho}_{k} \in H^{1}([0, l], \mathrm{SE}(3))$ has, first of all, a weakly converging subsequence with limit $\boldsymbol{\rho}_{*}$ only in $H^{1}\left([0, l], \mathbb{R}^{3} \times \mathbb{R}^{3 \times 3}\right)$. We have to show that this limit is in $H_{D, l}^{1}([0, l], \mathrm{SE}(3))$. By the compactness result of Rellich, weak convergence in $H^{1}\left([0, l], \mathbb{R}^{3} \times \mathbb{R}^{3 \times 3}\right)$ implies strong convergence $\boldsymbol{\rho}_{k} \rightarrow \boldsymbol{\rho}_{*}$ in $C\left([0, l], \mathbb{R}^{3} \times \mathbb{R}^{3 \times 3}\right)$. Since $\mathrm{SE}(3)$ is closed in $\mathbb{R}^{3} \times \mathbb{R}^{3 \times 3}$, we thus obtain $\boldsymbol{\rho}_{*}(s) \in \mathrm{SE}(3)$ for all $s \in[0, l]$. This shows our first assertion.

For our second assertion consider a weakly converging subsequence $\left(\phi_{k}, \boldsymbol{\rho}_{k}\right) \rightarrow$ $\left(\boldsymbol{\phi}_{*}, \boldsymbol{\rho}_{*}\right)$ in $\mathbf{H}^{1}(\mathcal{B}) \times H_{D, l}^{1}([0, l], \mathrm{SE}(3))$. By continuity and linearity of the trace operators $\left.(\cdot)\right|_{\Gamma}$ and $\left.(\cdot)\right|_{\gamma}$ this implies also $\left(\left.\phi_{k}\right|_{\Gamma},\left.\boldsymbol{\rho}_{k}\right|_{\gamma}\right) \rightarrow\left(\left.\phi_{*}\right|_{\Gamma},\left.\boldsymbol{\rho}_{*}\right|_{\gamma}\right)$. Thus, due to the weak closedness results of Lemma 4.1 and Lemma 4.3 we obtain that $F$ is weakly closed. Hence, as the indicator functional of a weakly closed set, $\iota_{F}$ is weakly lower semi-continuous.

Lemma 5.2. For $\phi \in \mathbf{H}^{1}(\mathcal{B})$ define the componentwise average on $\Gamma$ by

$$
\bar{\phi}_{\Gamma}:=\left.|\Gamma|^{-1} \int_{\Gamma} \phi\right|_{\Gamma} d A
$$


Then the Poincaré-type inequality

$$
\|\phi\|_{\mathbf{L}_{2}(\mathcal{B})} \leq\|\nabla \phi\|_{\mathbf{L}_{2}(\mathcal{B})}+\eta\left|\bar{\phi}_{\Gamma}\right|
$$

holds for some $\eta>0$.

Proof. Interpret (46) as a vector-valued linear functional on $H^{1}(\mathcal{B})$. This functional does not vanish on the constant functions. Then [11, Lem. B.63] asserts (47).

We use the previous definitions to write the constrained minimization problem as a nonsmooth unconstrained problem for the energy

$$
\begin{aligned}
\mathcal{E} & : \mathbf{H}^{1}(\mathcal{B}) \times H_{D, l}^{1}([0, l], \operatorname{SE}(3)) \rightarrow \overline{\mathbb{R}}, \\
\mathcal{E}(\boldsymbol{\phi}, \boldsymbol{\rho}) & =E(\boldsymbol{\phi})+j(\boldsymbol{\rho})+\iota_{F}(\boldsymbol{\phi}, \boldsymbol{\rho}) .
\end{aligned}
$$

This allows to state the main result of this section.

Theorem 5.1. The energy functional $\mathcal{E}$ has a global minimizer in $\mathbf{H}^{1}(\mathcal{B}) \times$ $H_{D, l}^{1}([0, l], \operatorname{SE}(3))$.

Proof. As a sum of weakly lower semi-continuous functionals, $\mathcal{E}$ is weakly lower semi-continuous as well.

Let us now show coercivity of $\mathcal{E}$. By Lemma 2.2 we know that $j$ is coercive in $H_{D, l}^{1}([0, l], \mathrm{SE}(3))$. Here, the Dirichlet boundary condition at $s=l$ is crucial. By the Sobolev embedding (8) we also get coercivity of $j$ in $C([0, l], \operatorname{SE}(3))$ and thus, in particular, $\left.\boldsymbol{\rho}\right|_{\gamma} \rightarrow \infty$ implies $j \rightarrow \infty$.

Further, by Assumption 3.1 $E$ is coercive in the semi-norm $\|\nabla \cdot\|_{\mathbf{L}_{2}(\mathcal{B})}$. By the Poincaré inequality 47 we have

$$
\|\phi\|_{\mathbf{H}^{1}(\mathcal{B})} \leq \eta\left(\|\nabla \phi\|_{\mathbf{L}_{2}(\mathcal{B})}+\left|\bar{\phi}_{\Gamma}\right|\right) \leq \eta\left(E(\phi)+\left|\bar{\phi}_{\Gamma}\right|\right) .
$$

Hence, it remains to prove that $\left|\bar{\phi}_{\Gamma}\right| \rightarrow \infty$ implies $\mathcal{E} \rightarrow \infty$. Here we have to exploit the coupling conditions. Both conditions imply (see (30) and (33)) that (writing $\left.\boldsymbol{\rho}\right|_{\gamma}=(r, R)$ )

$0=\int_{\Gamma}\left(\left.\phi\right|_{\Gamma}-r\right)-R R_{0}^{-1}\left(\left.\phi_{0}\right|_{\Gamma}-r_{0}\right) d A=|\Gamma|\left(\bar{\phi}_{\Gamma}-r\right)+\int_{\Gamma} R R_{0}^{-1}\left(\left.\phi_{0}\right|_{\Gamma}-r_{0}\right) d A$,

where the last integral is bounded independently of $R$, because $R \in \mathrm{SO}(3)$. Hence, $\left|\bar{\phi}_{\Gamma}\right| \rightarrow \infty$ implies $|r| \rightarrow \infty$, and thus in turn $j \rightarrow \infty$. This shows coercivity of $\mathcal{E}$.

Consider a minimizing sequence $\left(\phi_{k}, \boldsymbol{\rho}_{k}\right)$. By coercivity we conclude that $\phi_{k}$ is bounded in the Hilbert space $\mathbf{H}^{1}(\mathcal{B})$, and $\boldsymbol{\rho}_{k}$ is bounded in $H_{D, l}^{1}([0, l], \operatorname{SE}(3))$. Hence, by Lemma 5.1 we obtain a weakly converging subsequence with limit $\left(\phi_{*}, \boldsymbol{\rho}_{*}\right)$. Since $\left(\boldsymbol{\phi}_{k}, \boldsymbol{\rho}_{k}\right)$ is a minimizing sequence, we know that $\mathcal{E}\left(\boldsymbol{\phi}_{k}, \boldsymbol{\rho}_{k}\right) \rightarrow$ $\inf \mathcal{E}$. Since $\mathcal{E}$ is weakly lower semi-continuous (Lemma 5.1) we can conclude that

$$
\inf \mathcal{E} \leq \mathcal{E}\left(\phi_{*}, \boldsymbol{\rho}_{*}\right) \leq \lim _{k \rightarrow \infty} \mathcal{E}\left(\phi_{k}, \boldsymbol{\rho}_{k}\right)=\inf \mathcal{E},
$$

and hence $\mathcal{E}\left(\phi_{*}, \boldsymbol{\rho}_{*}\right)=\inf \mathcal{E}$. Thus, $\left(\phi_{*}, \boldsymbol{\rho}_{*}\right)$ is a minimizer of $\mathcal{E}$. 
Theorem 5.1 asserts the existence of a global minimizer. Since the energy functional usually is not convex, as for example in the case of nonlinear elasticity, this minimizer may be non-unique, which also reflects physical reality. The best one can hope for is local uniqueness, for which certain second order sufficient optimality conditions would have to be imposed.

Remark 5.1. We may easily extend our analysis to an arbitrary number of finitely many continua, rods, and couplings. Then our combined functional is a sum

$$
\mathcal{E}=\sum_{i=1}^{n_{E}} E_{i}\left(\boldsymbol{\phi}_{i}\right)+\sum_{k=1}^{n_{j}} j_{k}\left(\boldsymbol{\rho}_{k}\right)+\sum_{l=1}^{n_{c}} \iota_{F_{l}}\left(\boldsymbol{\phi}_{i(l)}, \boldsymbol{\rho}_{k(l)}\right) .
$$

In this more general setting, $\mathcal{E}$ is coercive if and only if there is a Dirichlet boundary condition for each connected component of the model.

\section{First order optimality conditions and weak formulations}

In this section we rigorously derive the first order necessary conditions for energy minimizers of our coupled problem. Existence of such minimizers has been shown in the previous section. Let in the following $\left(\phi_{*}, \boldsymbol{\rho}_{*}\right)$ be a local minimizer of $\mathcal{E}$, as defined in 48 , equipped with either rigid or averaged coupling conditions.

In Section 5 we have not used any assumptions on the smoothness of $j$ and $E$ at a minimizer. To derive the first-order optimality conditions we require differentiability of these functionals there.

Assumption 6.1. The continuum energy $E$ is Fréchet differentiable at $\phi_{*}$ with respect to perturbations in $\mathbf{C}^{1}(\mathcal{B})$, with derivative $T_{\phi_{*}} E \in \mathbf{H}^{1}(\mathcal{B})^{*}$. The rod energy $j$ is Fréchet differentiable at $\boldsymbol{\rho}_{*}$ in $C_{D, l}^{1}([0, l], \mathrm{SE}(3))$ with derivative $T_{\boldsymbol{\rho}_{*}} j \in Y_{\boldsymbol{\rho}_{*}, D, l}^{*}(c f .(16)$ ).

By our use of $C^{1}$-spaces we can include stored energy functionals with singularities, used for example in nonlinear elasticity to exclude local self-penetration [9].

If $j$ and $E$ are both quadratic functionals of the strains, then these spaces can be replaced by their $H^{1}$-counterparts. In this case, the following proofs could be simplified to some degree, but realistic stored energy functionals would be excluded. Still, Assumption 6.1 can usually not be verified a-priori for realistic stored energy functionals (cf. [2]), but only under the assumption that $\operatorname{det} \nabla \phi_{*}$ is bounded away from zero on $\mathcal{B}$.

In the following results, $c$ will mean either the rigid-coupling function $c_{\mathrm{r}}$ or the averaged-coupling function $c_{\mathrm{a}}(38)$.

Lemma 6.1. Let $\left(\phi_{*}, \boldsymbol{\rho}_{*}\right)$ be a minimizer of $\mathcal{E}$ and

$$
(\delta \phi, \delta \boldsymbol{\rho}) \in \mathbf{H}^{1}(\mathcal{B}) \times Y_{\boldsymbol{\rho}_{*}, D, l}
$$

such that

$$
T_{\left(\left.\boldsymbol{\phi}_{*}\right|_{\Gamma},\left.\boldsymbol{\rho}_{*}\right|_{\gamma}\right)} c\left(\left.\delta \boldsymbol{\phi}\right|_{\Gamma},\left.\delta \boldsymbol{\rho}\right|_{\gamma}\right)=0
$$

Suppose that Assumption 6.1 holds. Then

$$
T_{\boldsymbol{\phi}_{*}} E \delta \boldsymbol{\phi}+T_{\boldsymbol{\rho}_{*}} j \delta \boldsymbol{\rho}=0 .
$$


Proof. Since we can interpret $\boldsymbol{\rho}_{*}$ as the solution of a Dirichlet problem with $\boldsymbol{\rho}_{*}(l)$ and $\boldsymbol{\rho}_{*}(0)$ given, we can conclude by Theorem 2.1 that $\boldsymbol{\rho}_{*} \in C^{2}([0, l], \mathrm{SE}(3))$.

Consider a $C^{1}$-neighborhood $U$ of $\left(\phi_{*}, \boldsymbol{\rho}_{*}\right)$ in $\mathbf{H}^{1}(\mathcal{B}) \times C_{D, l}^{1}([0, l], \mathrm{SE}(3))$, and a local chart $\theta$ of $U$ into $\mathbf{C}^{1}(\mathcal{B}) \times\left(Y_{\boldsymbol{\rho}_{*}, D, l} \cap C^{1}\left([0, l], \mathbb{R}^{3} \times \mathbb{R}^{3 \times 3}\right)\right)$. Assume w.l.o.g. that $\theta\left(\boldsymbol{\phi}_{*}, \boldsymbol{\rho}_{*}\right)=(0,0)$.

In order to stay in a Hilbert space context we consider small local perturbations

$$
\begin{aligned}
\left(\boldsymbol{\phi}_{\theta}, \boldsymbol{\rho}_{\theta}\right) \in \mathbf{H}^{3}(\mathcal{B}) \times\left(Y_{\boldsymbol{\rho}_{*}, D, l} \cap H^{2}\left([0, l], \mathbb{R}^{3} \times \mathbb{R}^{3 \times 3}\right)\right) \\
\hookrightarrow \mathbf{C}^{1}(\mathcal{B}) \times\left(Y_{\boldsymbol{\rho}_{*}, D, l} \cap C^{1}\left([0, l], \mathbb{R}^{3} \times \mathbb{R}^{3 \times 3}\right)\right.
\end{aligned}
$$

and their corresponding preimages

$$
(\boldsymbol{\phi}, \boldsymbol{\rho}):=\theta^{-1}\left(\boldsymbol{\phi}_{\theta}, \boldsymbol{\rho}_{\theta}\right) \in \mathbf{H}^{1}(\mathcal{B}) \times H_{D, l}^{1}([0, l], \mathrm{SE}(3)),
$$

which lie in our $C^{1}$-neighborhood $U$. Then we obtain the local representations

$$
\begin{aligned}
(E+j)_{\theta}\left(\phi_{\theta}, \boldsymbol{\rho}_{\theta}\right) & :=E(\phi)+j(\boldsymbol{\rho}), \\
c_{\theta}\left(\phi_{\theta}, \boldsymbol{\rho}_{\theta}\right) & :=c\left(\left.\phi\right|_{\Gamma},\left.\boldsymbol{\rho}\right|_{\gamma}\right) .
\end{aligned}
$$

Thus, $c_{\theta}$ is defined as the local representation of the composition of $c$ and the trace mappings. We conclude that $c_{\theta}(0,0)=c\left(\left.\phi_{*}\right|_{\Gamma},\left.\boldsymbol{\rho}_{*}\right|_{\gamma}\right)=0$, and that $(0,0)$ is a local minimizer of the problem

$$
\min (E+j)_{\theta}\left(\phi_{\theta}, \boldsymbol{\rho}_{\theta}\right) \quad \text { on } \mathbf{H}^{3}(\mathcal{B}) \times\left(Y_{\boldsymbol{\rho}_{*}, D, l} \cap H^{2}\left([0, l], \mathbb{R}^{3} \times \mathbb{R}^{3 \times 3}\right)\right),
$$

subject to

$$
c_{\theta}\left(\phi_{\theta}, \boldsymbol{\rho}_{\theta}\right)=0 .
$$

Unlike the original problem the coordinate formulation is now posed in a linear space. By Lemmas 4.2 and 4.4 we know that $c_{\theta}$ is differentiable, and $(E+j)_{\theta}$ is differentiable by Assumption 6.1. Let us denote their derivatives by $(E+j)_{\theta}^{\prime}$ and $c_{\theta}^{\prime}$, respectively. To derive the equality 49 we will show that

$$
(E+j)_{\theta}^{\prime}(0,0)\left(\phi_{\theta}, \boldsymbol{\rho}_{\theta}\right) \leq 0,
$$

for all $\left(\phi_{\theta}, \boldsymbol{\rho}_{\theta}\right) \in \operatorname{ker} c_{\theta}^{\prime}(0,0)$. To achieve this, we first construct a local diffeomorphism $\Psi$ between $\operatorname{ker} c_{\theta}^{\prime}(0,0)$ and the set $c_{\theta}=0$ near $(0,0)$. We will use the surjective implicit function theorem [23, Thm.4.H]. For this, we need surjectivity of $c_{\theta}^{\prime}(0,0)$. In the case of rigid coupling,

$$
c_{\theta}^{\prime}=\left(c_{\mathrm{r}}\right)_{\theta}^{\prime}: \mathbf{H}^{3}(\mathcal{B}) \times\left(Y_{\boldsymbol{\rho}_{*}, D, l} \cap H^{2}\left([0, l], \mathbb{R}^{3} \times \mathbb{R}^{3 \times 3}\right)\right) \rightarrow \mathbf{H}^{5 / 2}(\Gamma)
$$

is surjective directly due to assumption (23), while the surjectivity of

$$
c_{\theta}^{\prime}=\left(c_{\mathrm{a}}\right)_{\theta}^{\prime}: \mathbf{H}^{3}(\mathcal{B}) \times\left(Y_{\boldsymbol{\rho}_{*}, D, l} \cap H^{2}\left([0, l], \mathbb{R}^{3} \times \mathbb{R}^{3 \times 3}\right)\right) \rightarrow\left(\mathbb{R}^{3} \times \mathbb{R}^{3 \times 3}\right)^{*}
$$

in the case of averaged coupling is due to Lemma 4.4 and $(23)$. Taking into account that the domain of $c_{\theta}^{\prime}$ is a Hilbert space (and thus its kernel has a closed complement), we can apply the surjective implicit function theorem to establish existence of a diffeomorphism $\Psi$ between $\operatorname{ker} c_{\theta}^{\prime}(0,0)$ and the set $c_{\theta}=0$ near $(0,0)$ in $\mathbf{H}^{3}(\mathcal{B}) \times\left(Y_{\boldsymbol{\rho}_{*}, D, l} \cap H^{2}\left([0, l], \mathbb{R}^{3} \times \mathbb{R}^{3 \times 3}\right)\right)$. This diffeomorphism can 
be chosen in a way such that $\Psi^{\prime}(0,0)$ is the identity. By differentiability of $(E+j)_{\theta}$ and $\Psi$, and the minimizing property of $(0,0)$ we conclude that

$$
0 \geq \lim _{t \rightarrow 0} \frac{(E+j)_{\theta}\left(\Psi\left(t \boldsymbol{\phi}_{\theta}, t \boldsymbol{\rho}_{\theta}\right)\right)-(E+j)_{\theta}(0,0)}{t}=(E+j)_{\theta}^{\prime}(0,0)\left(\boldsymbol{\phi}_{\theta}, \boldsymbol{\rho}_{\theta}\right),
$$

which is 500 .

The converse inequality can then be shown by inserting $\left(-\phi_{\theta},-\boldsymbol{\rho}_{\theta}\right)$. Since our result is invariant under changes of charts, we may switch to tangent vectors and tangent spaces by taking equivalence classes and obtain

$$
T_{\boldsymbol{\phi}_{*}} E \delta \phi+T_{\boldsymbol{\rho}_{*}} j \delta \boldsymbol{\rho}=0
$$

for all

$$
(\delta \phi, \delta \boldsymbol{\rho}) \in \mathbf{H}^{3}(\mathcal{B}) \times\left(Y_{\boldsymbol{\rho}_{*}, D, l} \cap H^{2}\left([0, l], \mathbb{R}^{3} \times \mathbb{R}^{3 \times 3}\right)\right)
$$

such that $\left(\left.\delta \boldsymbol{\phi}\right|_{\Gamma},\left.\delta \boldsymbol{\rho}\right|_{\gamma}\right) \in K_{\boldsymbol{\phi}_{*}, \boldsymbol{\rho}_{*}}$, where $K_{\boldsymbol{\phi}_{*}, \boldsymbol{\rho}_{*}}$ is either $K_{\boldsymbol{\phi}_{*}, \boldsymbol{\rho}_{*}}^{\mathrm{r}}$ (31) or $K_{\boldsymbol{\phi}_{*}, \boldsymbol{\rho}_{*}}^{\mathrm{a}} 42$.

Equation (51) is the desired assertion (49), but for a smaller set of test functions. To show (49) we have to extend (51) from the dense subspace $\mathbf{H}^{3}(\mathcal{B}) \times$ $\left(Y_{\boldsymbol{\rho}_{*}, D, l} \cap H^{2}\left([0, l], \mathbb{R}^{3} \times \mathbb{R}^{3 \times 3}\right)\right)$ of $\mathbf{H}^{1}(\mathcal{B}) \times Y_{\boldsymbol{\rho}_{*}, D, l}$ to the whole space. For this we need that the intersection of $K_{\phi_{*}, \rho_{*}}$ and the smoother space is dense in $K_{\phi_{*}, \rho_{*}}$. This has been shown in Proposition 4.1 for the rigid coupling and in Corollary 4.1 for the averaged coupling.

By Assumption 6.1 $T_{\boldsymbol{\phi}_{*}} E$ and $T_{\boldsymbol{\rho}_{*}} j$ are continuous on $\mathbf{H}^{1}(\mathcal{B}) \times Y_{\boldsymbol{\rho}_{*}, D, l}$, and $T_{\left(\boldsymbol{\phi}_{*}, \boldsymbol{\rho}_{*}\right)}(E+j)$ is continuous on this space, too. By these density and continuity assumptions, we can perform the desired extension by a simple approximation argument and passing to the limit.

We can now derive the first-order optimality conditions.

Theorem 6.1. Set $V$ as in Chapter 4, i.e., $V=\mathbf{H}^{1 / 2}(\Gamma)$ if $c=c_{r}$ (rigid coupling), and $V=\mathbb{R}^{3} \times \mathbb{R}^{3}$ if $c=c_{a}$ (averaged coupling). Suppose that Assumption 6.1 holds.

There exists a Lagrange multiplier $\lambda \in V^{*}$ such that the equation

$$
T_{\boldsymbol{\phi}_{*}} E \delta \boldsymbol{\phi}+T_{\boldsymbol{\rho}_{*}} j \delta \boldsymbol{\rho}-\lambda T_{\left(\left.\boldsymbol{\phi}_{*}\right|_{\Gamma},\left.\boldsymbol{\rho}_{*}\right|_{\gamma}\right)} c\left(\left.\delta \phi\right|_{\Gamma},\left.\delta \boldsymbol{\rho}\right|_{\gamma}\right)=0
$$

is satisfied for all $(\delta \boldsymbol{\phi}, \delta \boldsymbol{\rho}) \in \mathbf{H}^{1}(\mathcal{B}) \times Y_{\boldsymbol{\rho}_{*}, D, l}$.

Proof. Define $K_{\phi_{*}, \rho_{*}}$ by either (31) or (42), and consider the functional

$$
F(\delta \phi, \delta \boldsymbol{\rho}):=T_{\phi_{*}} E \delta \phi+T_{\boldsymbol{\rho}_{*}} j \delta \boldsymbol{\rho}+\iota_{K_{\phi_{*}, \boldsymbol{\rho}_{*}}}(\delta \boldsymbol{\phi}, \delta \boldsymbol{\rho}) .
$$

We apply the rules of subdifferential calculus [10, Sec.I.5] to this functional. Recall that the subdifferential of a functional $f: X \rightarrow \overline{\mathbb{R}}$ at a point $x_{0} \in X$ is defined by

$$
\partial f\left(x_{0}\right):=\left\{\ell \in X^{*} \mid \ell\left(x-x_{0}\right) \leq f(x)-f\left(x_{0}\right) \quad \forall x \in X\right\} .
$$

By 49 we have $F=0$ on $K_{\boldsymbol{\phi}_{*}, \boldsymbol{\rho}_{*}}$ and $+\infty$ otherwise. Hence,

$$
0 \in \partial F(0,0)=\partial\left(T_{\boldsymbol{\phi}_{*}} E+T_{\boldsymbol{\rho}_{*}} j+\iota_{K_{\boldsymbol{\phi}_{*}, \boldsymbol{\rho}_{*}}}\right)(0,0) .
$$


Since $T_{\boldsymbol{\phi}_{*}} E$ and $T_{\boldsymbol{\rho}_{*}} j$ are linear and continuous in $\mathbf{H}^{1}(\mathcal{B}) \times Y_{\boldsymbol{\rho}_{*}, D, l}$, application of the sum-rule of subdifferential calculus [10, Prop. I.5.6] provides the existence of an $\ell \in \partial \iota_{K_{\phi_{*}, \boldsymbol{\rho}_{*}}}(0,0)$ such that

$$
0=T_{\phi_{*}} E+T_{\boldsymbol{\rho}_{*}} j+\ell .
$$

It is easy to see that the subdifferential of an indicator function of a closed linear subspace can be characterized as its annihilator, i.e.,

$$
\partial \iota_{K_{\boldsymbol{\phi}_{*}, \boldsymbol{\rho}_{*}}}(0,0)=K_{\boldsymbol{\phi}_{*}, \boldsymbol{\rho}_{*}}^{\perp}=\left\{\ell \in \mathbf{H}^{1}(\mathcal{B})^{*} \times Y_{\boldsymbol{\rho}_{*}^{*}, D, l}^{*} \mid \ell(x)=0 \forall x \in K_{\boldsymbol{\phi}_{*}, \boldsymbol{\rho}_{*}}\right\} .
$$

Let us define the linear operator $A$ as the composition of $T_{\left(\phi_{*}, \rho_{*}\right)} c$ and the trace operators, via $A(\delta \boldsymbol{\phi}, \delta \boldsymbol{\rho}):=T_{\left(\boldsymbol{\phi}_{*}, \rho_{*}\right)} c\left(\left.\delta \phi\right|_{\Gamma},\left.\delta \boldsymbol{\rho}\right|_{\gamma}\right)$, which is continuous and surjective as a composition of continuous and surjective operators. By definition ker $A=K_{\boldsymbol{\phi}_{*}, \boldsymbol{\rho}_{*}}$. Application of the closed range theorem [5, Thm. 2.19] yields

$$
K_{\boldsymbol{\phi}_{*}, \boldsymbol{\rho}_{*}}^{\perp}=(\operatorname{ker} A)^{\perp}=\operatorname{ran}\left(A^{*}\right),
$$

for the adjoint $A^{*}$ of $A$. Hence, since $\ell \in K_{\boldsymbol{\phi}_{*}, \boldsymbol{\rho}_{*}}^{\perp}=\operatorname{ran}\left(A^{*}\right)$, there exists a $\lambda \in V^{*}$ such that

$$
\ell=-A^{*} \lambda
$$

(the minus sign is chosen deliberately), which means that

$$
\ell(\delta \boldsymbol{\phi}, \delta \boldsymbol{\rho})=-\lambda T_{\left(\boldsymbol{\phi}_{*}, \rho_{*}\right)} c\left(\left.\delta \boldsymbol{\phi}\right|_{\Gamma},\left.\delta \boldsymbol{\rho}\right|_{\gamma}\right) \quad \forall(\delta \boldsymbol{\phi}, \delta \boldsymbol{\rho}) \in \mathbf{H}^{1}(\mathcal{B}) \times Y_{\boldsymbol{\rho}_{*}, D, l} .
$$

Inserting this into 53 proves our assertion.

Equation $(52)$ is the first-order optimality condition for our coupled problem, both with rigid and averaged coupling conditions. Splitting of $(52)$ into the components corresponding to elastic body and rod yields the following alternative representation

$$
\begin{array}{rlrl}
T_{\boldsymbol{\phi}_{*}} E \delta \boldsymbol{\phi}-\lambda T_{\left(\left.\boldsymbol{\phi}_{*}\right|_{\Gamma},\left.\boldsymbol{\rho}_{*}\right|_{\gamma}\right)} c\left(\left.\delta \boldsymbol{\phi}\right|_{\Gamma}, 0\right) & =0 & & \forall \delta \boldsymbol{\phi} \in \mathbf{H}^{1}(\mathcal{B}) \\
T_{\boldsymbol{\rho}_{*}} j \delta \boldsymbol{\rho}-\lambda T_{\left(\left.\boldsymbol{\phi}_{*}\right|_{\Gamma},\left.\boldsymbol{\rho}_{*}\right|_{\gamma}\right)} c\left(0,\left.\delta \boldsymbol{\rho}\right|_{\gamma}\right) & =0 & & \forall \delta \boldsymbol{\rho} \in Y_{\boldsymbol{\rho}_{*}, D, l} \\
c\left(\left.\boldsymbol{\phi}_{*}\right|_{\Gamma},\left.\boldsymbol{\rho}_{*}\right|_{\gamma}\right) & =0 &
\end{array}
$$

We have chosen the negative sign in front of $\lambda$ in order to make $\lambda$ easier to interpret as a boundary force in the following section.

\section{Coupling conditions for the dual variables}

Theorem 6.1 allows to derive coupling conditions for the dual variables. These conditions can be interpreted in terms of forces and moments. Unlike in the previous chapters we treat rigid and averaged coupling separately again.

\subsection{Rigid coupling}

In the case of rigid coupling, the codomain of $c_{\mathrm{r}}$ is the infinite dimensional space $\mathbf{H}^{1 / 2}(\Gamma)$. Thus, we will obtain boundary stresses in $\mathbf{H}^{1 / 2}(\Gamma)^{*}$. 
Proposition 7.1 (Dual conditions). Suppose that $\left(\boldsymbol{\phi}_{*}, \boldsymbol{\rho}_{*}\right)$ satisfies the first order optimality conditions (52) with $c=c_{r}$, and is sufficiently smooth. Let $(r, R)=\left.\boldsymbol{\rho}_{*}\right|_{\gamma}$. Then the dual conditions

$$
\begin{aligned}
\left.\mathbf{n}\right|_{\gamma} & =\int_{\Gamma} P \boldsymbol{\nu}(x) d A \\
\left.\mathbf{m}\right|_{\gamma} & =\int_{\Gamma}\left(\left.\phi_{*}\right|_{\Gamma}(x)-r\right) \times P \boldsymbol{\nu}(x) d A
\end{aligned}
$$

hold, and $P \boldsymbol{\nu}=\lambda$ on $\Gamma$.

Proof. Inserting the definitions of $T_{\boldsymbol{\phi}_{*}} E$ (Eq. 22 ) and $T_{\left(\left.\boldsymbol{\phi}_{*}\right|_{\Gamma},\left.\boldsymbol{\rho}_{*}\right|_{\gamma}\right)} c_{\mathrm{r}}$ (given in Lemma 4.2 into 54a we obtain

$$
\begin{aligned}
\int_{\mathcal{B}} P\left(\nabla \phi_{*}\right): & \nabla \delta \phi-f \cdot \delta \phi d V \\
& -\left.\int_{\partial_{N} \mathcal{B}} g \cdot \delta \phi\right|_{\partial_{N} \mathcal{B}} d A-\left\langle\lambda,\left.\delta \phi\right|_{\Gamma}\right\rangle_{\mathbf{H}^{1 / 2}(\Gamma)}=0 \quad \forall \delta \boldsymbol{\phi} \in \mathbf{H}^{1}(\mathcal{B}) .
\end{aligned}
$$

Formal integration by parts yields Neumann boundary conditions

$$
P \boldsymbol{\nu}=\lambda \text { on } \Gamma, \quad \text { and } \quad P \boldsymbol{\nu}=g \text { on } \partial_{N} \mathcal{B}
$$

where $\lambda$ is an element of $\mathbf{H}^{1 / 2}(\Gamma)^{*}$. Thus, $\lambda$ can be interpreted as a boundary force on $\Gamma$.

We will assume in the following that $\lambda$ is an integrable function, which holds if $\boldsymbol{\phi}_{*}$ is sufficiently smooth. Plugging the definition of $T_{\left(\left.\boldsymbol{\phi}_{*}\right|_{\Gamma},\left.\boldsymbol{\rho}_{*}\right|_{\gamma}\right)} c_{\mathrm{r}}$ given in Lemma 4.2 into $54 \mathrm{~b}$ yields

$$
T_{\boldsymbol{\rho}_{*}} j \delta \boldsymbol{\rho}-\int_{\Gamma} \lambda \cdot\left(-\delta r-\delta R R_{0}^{-1}\left(\left.\phi_{0}\right|_{\Gamma}-r_{0}\right)\right) d A=0 \quad \forall \delta \boldsymbol{\rho} \in Y_{\boldsymbol{\rho}_{*}, D, l},
$$

with $(\delta r, \delta R)=\left.\delta \boldsymbol{\rho}\right|_{\gamma}$. Writing $\lambda T_{\left(\left.\boldsymbol{\phi}_{*}\right|_{\Gamma},\left.\boldsymbol{\rho}_{*}\right|_{\gamma}\right)} c_{\mathrm{r}}=b^{*}(\delta r)+B^{*}(\delta R)$ we can use Theorem 2.2 with

$b^{*}(\delta r)=-\int_{\Gamma} \lambda \cdot \delta r d A \quad$ and $\quad B^{*}(\delta R)=-\int_{\Gamma} \lambda \cdot\left(\delta R R_{0}^{-1}\left(\left.\phi_{0}\right|_{\Gamma}-r_{0}\right)\right) d A$.

In particular, from follows

$$
\left.\mathbf{n}^{*}\right|_{\gamma}(\delta r)=-b^{*}(\delta r)=\int_{\Gamma} \lambda \cdot \delta r d A=\int_{\Gamma} \lambda d A \cdot \delta r,
$$

i.e.,

$$
\left.\mathbf{n}\right|_{\gamma}=\int_{\Gamma} \lambda d A=\int_{\Gamma} P \boldsymbol{\nu}(x) d A .
$$

For the moments $\left.\mathbf{m}\right|_{\gamma}$ we obtain, using 30

$$
-B^{*}(\delta R)=\int_{\Gamma} \lambda \cdot \delta R R_{0}^{-1}\left(\left.\phi_{0}\right|_{\Gamma}-r_{0}\right) d A=\int_{\Gamma} \lambda \cdot\left(\delta R R^{-1}\right)\left(\left.\phi_{*}\right|_{\Gamma}-r\right) d A .
$$


We rewrite this expression in terms of $\delta w:=\left(\delta R R^{-1}\right)_{\times}$. Using (4) we obtain

$$
\begin{aligned}
\left.\mathbf{m}^{*}\right|_{\gamma}(\delta w) & =\int_{\Gamma} \lambda \cdot\left(\delta R R^{-1}\right)\left(\left.\phi_{*}\right|_{\Gamma}-r\right) d A=\int_{\Gamma} \lambda \cdot\left(\delta w \times\left(\left.\phi_{*}\right|_{\Gamma}-r\right)\right) d A \\
& =\int_{\Gamma} \delta w \cdot\left(\left(\left.\phi_{*}\right|_{\Gamma}-r\right) \times \lambda\right) d A=\int_{\Gamma}\left(\left.\phi_{*}\right|_{\Gamma}-r\right) \times \lambda d A \cdot \delta w .
\end{aligned}
$$

Thus, we can identify

$$
\left.\mathbf{m}\right|_{\gamma}=\int_{\Gamma}\left(\left.\phi_{*}\right|_{\Gamma}-r\right) \times \lambda d A=\int_{\Gamma}\left(\left.\phi_{*}\right|_{\Gamma}-r\right) \times P \boldsymbol{\nu}(x) d A .
$$

As mentioned, $\lambda \in \mathbf{H}^{1 / 2}(\Gamma)^{*}$ can be interpreted as a normal stress density on the continuum coupling boundary $\Gamma$. The equations in Proposition 7.1 then signify equality of the total force and moment transmitted by the continuum across $\Gamma$ and by the rod across the cross-section at $\gamma$.

\subsection{Averaged coupling}

In the case of average coupling, the codomain space of $c_{\mathrm{a}}$ is $\mathbb{R}^{3} \times \mathbb{R}^{3}$, so that the dual variables correspond to a force vector and a moment vector. Before we proceed, we prove the following technical lemma.

Lemma 7.1. Let $u \in \mathbb{R}^{3}$, and $v(x), w(x) \in \mathbf{L}_{2}(\Gamma)$ with $\int_{\Gamma} v(x) \times w(x) d x=0$. Then

$$
\int_{\Gamma} w(x) \times(u \times v(x)) d x=\int_{\Gamma} v(x) \times(u \times w(x)) d x .
$$

Proof. We can compute pointwise almost everywhere

$$
\begin{aligned}
w \times(u \times v)-v \times(u \times w) & =w \times(u \times v)+(u \times w) \times v \\
& =-u \times(v \times w),
\end{aligned}
$$

where the last equality is the Jacobi identity. Integration over $\Gamma$ yields

$\int_{\Gamma} w(x) \times(u \times v(x))-v(x) \times(u \times w(x)) d x=-u \times\left(\int_{\Gamma} v(x) \times w(x) d x\right)=0$,

which was asserted.

Proposition 7.2 (Dual conditions). Suppose that $\left(\phi_{*}, \boldsymbol{\rho}_{*}\right)$ satisfies the first order optimality conditions (52) with $c=c_{a}$, and is sufficiently smooth. Let $(r, R)=\left.\boldsymbol{\rho}_{*}\right|_{\gamma}$. Then the dual conditions

$$
\begin{aligned}
\left.\mathbf{n}\right|_{\gamma} & =\int_{\Gamma} P \boldsymbol{\nu} d A \\
\left.\mathbf{m}\right|_{\gamma} & =\int_{\Gamma}\left(\left.\phi_{*}\right|_{\Gamma}(x)-r\right) \times P \boldsymbol{\nu} d A,
\end{aligned}
$$

hold at the contact boundary, where P $\boldsymbol{\nu}$ is of the form

$$
P \boldsymbol{\nu}(x)=\lambda_{1}-\lambda_{2} \times R R_{0}^{-1}\left(\left.\phi_{0}\right|_{\Gamma}(x)-r_{0}\right)
$$

for some $\left(\lambda_{1}, \lambda_{2}\right) \in \mathbb{R}^{3} \times \mathbb{R}^{3}$. 
Proof. Let us denote again for brevity

$$
\psi: \Gamma \rightarrow \mathbb{R}^{3}, \quad \psi(x):=R_{0}^{-1}\left(\left.\phi_{0}\right|_{\Gamma}(x)-r_{0}\right) .
$$

The first line of the dual coupling conditions (54) is

$$
T_{\boldsymbol{\phi}_{*}} E \delta \boldsymbol{\phi}-\lambda T_{\left(\left.\boldsymbol{\phi}_{*}\right|_{\Gamma},\left.\boldsymbol{\rho}_{*}\right|_{\gamma}\right)} c\left(\left.\delta \boldsymbol{\phi}\right|_{\Gamma}, 0\right)=0 \quad \forall \delta \boldsymbol{\phi} \in \mathbf{H}^{1}(\mathcal{B}) .
$$

We write $\lambda=\left(\lambda_{1}, \lambda_{2}\right)$ with $\lambda_{1} \in \mathbb{R}^{3}$ and $\lambda_{2} \in \mathbb{R}^{3}$, and insert the definitions of $T_{\boldsymbol{\phi}_{*}} E($ Eq. 22) $)$ and $T_{\left(\left.\boldsymbol{\phi}_{*}\right|_{\Gamma},\left.\boldsymbol{\rho}_{*}\right|_{\gamma}\right)} c_{\mathrm{a}}(\mathrm{Eq} \cdot 39)$ to obtain

$$
\begin{aligned}
\int_{\mathcal{B}}\left(P\left(\nabla \phi_{*}\right):\right. & \nabla \delta \phi-f \cdot \delta \phi) d V-\int_{\partial_{N} \mathcal{B}} g \cdot \delta \phi d A \\
& -\left[\lambda_{1} \cdot \int_{\Gamma} \delta \phi d A+\lambda_{2} \cdot \int_{\Gamma} \delta \phi \times R \psi d A\right]=0 \quad \forall \delta \phi \in \mathbf{H}^{1}(\mathcal{B}) .
\end{aligned}
$$

The second term in the brackets is

$$
\lambda_{2} \cdot \int_{\Gamma}(\delta \phi \times R \psi) d A=\int_{\Gamma} \delta \phi \cdot\left(R \psi \times \lambda_{2}\right) d A=\int_{\Gamma}-\delta \phi \cdot\left(\lambda_{2} \times R \psi\right) d A,
$$

where we have used (4) and the anti-symmetry of the cross product. Together with the first term in the brackets, integration by parts yields

$$
P \boldsymbol{\nu}(x)=\lambda_{1}-\lambda_{2} \times R \psi(x)
$$

for almost all $x$ on $\Gamma$.

The second line of the optimality conditions is

$$
T_{\boldsymbol{\rho}_{*}} j \delta \boldsymbol{\rho}-\lambda T_{\left(\left.\boldsymbol{\phi}_{*}\right|_{\Gamma},\left.\boldsymbol{\rho}_{*}\right|_{\gamma}\right)} c_{\mathrm{a}}\left(0,\left.\delta \boldsymbol{\rho}\right|_{\gamma}\right)=0 \quad \forall \delta \boldsymbol{\rho} \in Y_{\boldsymbol{\rho}_{*}, D, l}
$$

Inserting the definition of $T_{\left(\left.\boldsymbol{\phi}_{*}\right|_{\Gamma},\left.\boldsymbol{\rho}_{*}\right|_{\gamma}\right)} c_{\mathrm{a}}$ from Lemma 4.4 we obtain

$$
\begin{aligned}
T_{\boldsymbol{\rho}_{*}} j \delta \boldsymbol{\rho} & -\lambda_{1} \cdot \int_{\Gamma}(-\delta r-\delta R \psi) d A \\
& -\lambda_{2} \cdot \int_{\Gamma}[(-\delta r) \times R \psi+(\boldsymbol{\phi}-r) \times \delta R \psi] d A=0 \quad \forall \delta \boldsymbol{\rho} \in Y_{\boldsymbol{\rho}_{*}, D, l},
\end{aligned}
$$

where we have again set $\lambda=\left(\lambda_{1}, \lambda_{2}\right)$. We write this as

$$
T_{\boldsymbol{\rho}_{*}} j \delta \boldsymbol{\rho}-\beta(\delta r, \delta R)=0 \quad \forall \delta \boldsymbol{\rho} \in Y_{\boldsymbol{\rho}_{*}, D, l},
$$

with $\beta(\delta r, \delta R)=b^{*}(\delta r)+B^{*}(\delta R)$,

$$
b^{*}(\delta r)=-\lambda_{1} \cdot \int_{\Gamma} \delta r d A-\lambda_{2} \cdot \int_{\Gamma} \delta r \times R \psi d A
$$

and

$$
B^{*}(\delta R)=-\lambda_{1} \cdot \int_{\Gamma} \delta R \psi d A+\lambda_{2} \cdot \int_{\Gamma}\left(\left.\phi_{*}\right|_{\Gamma}-r\right) \times \delta R \psi d A
$$


Using Theorem 2.2 we get with (4)

$$
\begin{aligned}
\left.\mathbf{n}\right|_{\gamma} \cdot \delta r & =-b^{*}(\delta r)=\lambda_{1} \cdot \int_{\Gamma} \delta r d A+\lambda_{2} \cdot \int_{\Gamma} \delta r \times R \psi d A \\
& =\lambda_{1} \cdot \int_{\Gamma} \delta r d A+\delta r \cdot \int_{\Gamma} R \psi \times \lambda_{2} d A=\int_{\Gamma} \delta r \cdot\left(\lambda_{1}-\lambda_{2} \times R \psi\right) d A .
\end{aligned}
$$

For the orientation part, Theorem 2.2 gives (using again $\delta w:=\left(\delta R R^{-1}\right)_{\times}$)

$$
\begin{aligned}
\left.\mathbf{m}\right|_{\gamma} \cdot \delta w & =-B^{*}(\delta R)=-B^{*}\left(\delta w^{\times} R\right) \\
& =\lambda_{1} \cdot \int_{\Gamma}(\delta w \times R \psi) d A-\lambda_{2} \cdot \int_{\Gamma}\left(\left.\phi_{*}\right|_{\Gamma}-r\right) \times(\delta w \times R \psi) d A .
\end{aligned}
$$

Consider the first term of the second line. We can use (4), the coupling conditions (33) and again (4) to compute

$$
\begin{aligned}
& \int_{\Gamma} \lambda_{1} \cdot(\delta w \times R \psi(x)) d A=\int_{\Gamma} R \psi(x) \cdot\left(\lambda_{1} \times \delta w\right) d A \\
&=\int_{\Gamma}\left(\left.\phi_{*}\right|_{\Gamma}-r\right) \cdot\left(\lambda_{1} \times \delta w\right) d A=\int_{\Gamma}\left(\left.\phi_{*}\right|_{\Gamma}-r\right) \times \lambda_{1} d A \cdot \delta w .
\end{aligned}
$$

For the second term we use (4) twice and Lemma 7.1, which applies due to (34) to compute

$$
\begin{aligned}
\lambda_{2} \cdot \int_{\Gamma}\left(\left.\phi_{*}\right|_{\Gamma}-r\right) \times(\delta w \times R \psi) d A & =\int_{\Gamma}(\delta w \times R \psi(x)) \cdot\left(\lambda_{2} \times\left(\left.\phi_{*}\right|_{\Gamma}-r\right)\right) d A \\
& =\int_{\Gamma} \delta w \cdot\left(R \psi(x) \times\left(\lambda_{2} \times\left(\left.\phi_{*}\right|_{\Gamma}-r\right)\right)\right) d A \\
& =\int_{\Gamma}\left(\left.\phi_{*}\right|_{\Gamma}-r\right) \times\left(\lambda_{2} \times R \psi(x)\right) d A \cdot \delta w .
\end{aligned}
$$

Adding both terms yields finally

$$
\left.\mathbf{m}^{*}\right|_{\gamma}(\delta w)=\int_{\Gamma}\left(\left.\phi_{*}\right|_{\Gamma}-r\right) \times\left(\lambda_{1}-\lambda_{2} \times R \psi(x)\right) d A \cdot \delta w,
$$

for all $\delta w \in \mathbb{R}^{3}$ and thus the desired result.

In contrast to the case of rigid coupling, the possible constraint forces given by (57) form a vector space of only six dimensions, parametrized by the Lagrange multipliers $\left(\lambda_{1}, \lambda_{2}\right)$.

To gain additional insight into these results, we consider the case that $r_{0}$ is at the center of gravity of $\phi_{0}(\Gamma)$. In this case $\lambda_{1}$ and $\lambda_{2}$ decouple. The vector $\lambda_{1}$ is then the average force density transmitted, and $\lambda_{2}$ is related to the average moment density.

Proposition 7.3. Assume that $r_{0}$ is the center of gravity of $\phi_{0}(\Gamma)$

$$
\int_{\Gamma}\left(\left.\phi_{0}\right|_{\Gamma}(x)-r_{0}\right) d A=0 .
$$

Then

$$
\begin{aligned}
\left.\mathbf{n}\right|_{\gamma} & =\int_{\Gamma} \lambda_{1} d A \\
\left.\mathbf{m}\right|_{\gamma} & =\int_{\Gamma}\left(\phi_{*}(x)-r\right) \times\left(-\lambda_{2} \times R R_{0}^{-1}\left(\phi_{0}(x)-r_{0}\right)\right) d A .
\end{aligned}
$$


Proof. Inserting (57) into 55) we obtain

$$
\left.\mathbf{n}\right|_{\gamma}=\int_{\Gamma}\left(\lambda_{1}-\lambda_{2} \times R R_{0}^{-1}\left(\left.\phi_{0}\right|_{\Gamma}(x)-r_{0}\right)\right) d A .
$$

Taking into account (58) we obtain 59 because $\lambda_{2} \times R R_{0}^{-1}$ is independent of $x$. Inserting (57) into (56) we get

$$
\left.\mathbf{m}\right|_{\gamma}=\int_{\Gamma}\left(\phi_{*}(x)-r\right) \times\left(\lambda_{1}-\lambda_{2} \times R R_{0}^{-1}\left(\left.\phi_{0}\right|_{\Gamma}(x)-r_{0}\right)\right) d A,
$$

where, due to 33 and 58

$$
\int_{\Gamma}\left(\left.\phi_{*}\right|_{\Gamma}(x)-r\right) d A=0 .
$$

Hence, 60 follows, since $\lambda_{1}$ is independent of $x$.

\section{Outlook}

In this article we have analyzed a coupled problem involving models of different dimensions and orientation degrees of freedom from a constraint minimization perspective. Under increasing regularity assumptions we arrived at a hierarchy of results: from energy minimizers via weak formulations to balance equations.

Our work can be extended in several directions. First of all, as already noted, it is straightforward to consider multiple rods and elastic continua, and multiple couplings. Further, in many situations of interest, there are also Signorini-type contact conditions present. In principle, our approach can be extended to this case. One would then have to replace the differentiability assumption on the energy functional of the elastic body by subdifferentiability. Dynamic coupling problems can be analyzed by a semi-group approach, where discretization in time leads to a sequence of stationary problems. Coupling of elastic continua to two-dimensional Cosserat shells is another interesting topic.

Our approach may also prove beneficial for solution algorithms. In principle, the problem (24) together with 25 can be treated directly with a constraint minimization algorithm, resulting in a "monolithic" method for the coupled problem. On the other hand, the availability of an energy formulation may help to analyze Steklov-Poincaré-type algorithms, or provide viable globalization strategies. This is a topic for future research.

\section{References}

[1] S. S. Antman. Nonlinear problems of elasticity, volume 107 of Applied mathematical sciences. Springer, 1991.

[2] J. M. Ball. Some open problems in elasticity. In P. Newton, P. Holmes, and A. Weinstein, editors, Geometry, Mechanics, and Dynamics, pages 3-59. Springer, 2002.

[3] F. Bethuel. The approximation problem for Sobolev maps between two manifolds. Acta Math., 167:153-206, 1991. 
[4] P. J. Blanco, M. Discacciati, and A. Quarteroni. Modeling dimensionallyheterogenenous problems: analysis, approximation and applications. $\mathrm{Nu}$ mer. Math., 119(2):299-335, 2011.

[5] H. Brezis. Functional Analysis, Sobolev Spaces and Partial Differential Equations. Springer, 2011.

[6] N. Chouaïeb. Kirchhoff's Problem of Helical Solutions of Uniform Rods and their Stability Properties. PhD thesis, Ecole Polytechnique Fédérale Lausanne, 2003.

[7] P. Ciarlet, H. LeDret, and R. Nzengwa. Junctions between threedimensional and two-dimensional linearly elastic structures. J. Math. Pures Appl., 68:261-295, 1989.

[8] P. G. Ciarlet. Mathematical Elasticity Vol. I: Three-dimensional Elasticity. North-Holland, 1988.

[9] P. G. Ciarlet. Mathematical Elasticity. North-Holland, 1988.

[10] I. Ekeland and R. Temam. Convex Analysis and Variational Problems. SIAM, 1999

[11] A. Ern and J.-L. Guermond. Theory and Practice of Finite Elements. Springer, 2004.

[12] L. Formaggia, J. Gerbeau, F. Nobile, and A. Quarteroni. On the coupling of 3D and 1D Navier-Stokes equations for flow problems in compliant vessels. Computer Methods in Applied Mechanics and Engineering, 191:561-582, 2001.

[13] H. Karcher. Riemannian center of mass and mollifier smoothing. Comm. Pure Appl. Math., 30:509-541, 1977.

[14] S. Kehrbaum. Hamiltonian Formulations of the Equilibrium Conditions Governing Elastic Rods: Qualitative Analysis and Effective Properties. $\mathrm{PhD}$ thesis, University of Maryland, 1997.

[15] J. Lagnese, G. Leugering, and E. Schmidt. Modeling, Analysis and Control of Dynamic Elastic Multi-Link Structures. Birkhäuser, 1994.

[16] D. J. Monaghan, I. W. Doherty, D. M. Court, and C. G. Armstrong. Coupling 1D beams to 3D bodies. In Proc. 7th Int. Meshing Roundtable. Sandia National Laboratories, 1998.

[17] R. S. Palais. Morse theory on Hilbert manifolds. Topology, 2:299-340, 1963.

[18] O. Sander. Multidimensional Coupling in a Human Knee Model. PhD thesis, Freie Universität Berlin, 2008.

[19] O. Sander. Coupling geometrically exact Cosserat rods and linear elastic continua. In Proc. of DD20, to appear.

[20] T. Seidman and P. Wolfe. Equilibrium states of an elastic conducting rod in a magnetic field. Arch. Rational Mech. Anal., 102(4):307-329, 1988. 
[21] R. Temam. Navier-Stokes equations: theory and numerical analysis. AMS, 1977.

[22] J. Wloka. Partielle Differentialgleichungen. Teubner-Verlag, 1982.

[23] E. Zeidler. Nonlinear Functional Analysis and its Applications, volume I. Springer, 1986. 